\title{
Communication and Authority with a Partially Informed Expert*
}

\author{
Murali Agastya $^{\dagger} \quad$ Parimal Kanti Bag ${ }^{\ddagger} \quad$ Indranil Chakraborty ${ }^{\S}$
}

March 12, 2012

\begin{abstract}
A partially informed expert, A, strategically transmits information to a principal, $\mathbf{P}$. The residual uncertainty faced by the expert effectively causes the bias between $\mathbf{P}$ and $\mathbf{A}$ to be random, with two consequences. First, by misreporting, $\mathbf{A}$ is likely to induce a decision choice by $\mathbf{P}$, after the resolution of the residual uncertainty, that is either close to A's ideal position or too far from it, whereas truthful reporting keeps such variations "more balanced". A convex loss function of A thus favors truthful reporting. Second, by retaining authority of decision making and communicating with $\mathbf{A}, \mathbf{P}$ avoids exposure to risks due to residual uncertainty. Better information transmission and the associated insurance benefit thus often imply $\mathbf{P}$ preferring control over delegation, despite $\mathbf{A}$ having superior information.

JEL Classification: C72, D82, D83.

Key Words: Authority, Delegation, Effective Bias, Full Revelation, Partially Informed Expert, Strategic Information Transmission, Multidimensional Uncertainty.
\end{abstract}

*We thank Bob Anderson, Ricardo Alonso, Bob Gibbons, Atsushi Kajii and Navin Kartik for their very helpful comments and conversations. This work has also benefited from presentations at various seminars and conferences.

†School of Economics, H04 Merewether Building, University of Sydney NSW 2006, Australia; Email: murali.agastya@sydney.edu.au

+Department of Economics, National University of Singapore, Faculty of Arts and Social Sciences, AS2 Level 6, 1 Arts Link, Singapore 117570; E-mail: ecsbpk@nus.edu.sg

${ }^{\S}$ Department of Economics, National University of Singapore, Faculty of Arts and Social Sciences, AS2 Level 6, 1 Arts Link, Singapore 117570; E-mail: indro@nus.edu.sg 


\section{Introduction}

In many economic situations where an action affects the welfare of two parties, the formal decision rights belong to one, say a receiver (Principal or $\mathbf{P}$ ) whereas relevant information lies with another, a sender (Agent or A). In many such settings, it is not possible to write binding contracts and construct payment schemes that are contingent on chosen actions. One possible avenue for $\mathbf{P}$ is to elicit information from $\mathbf{A}$ and then take a decision. However, as the seminal contribution Crawford and Sobel (1982) shows, the ability of $\mathbf{P}$ to elicit information from $\mathbf{A}$ to make an informed choice is limited as the latter strategically controls the information she transmits. Aghion and Tirole (1997) and Dessein (2002) explore another avenue - the value of $\mathbf{P}$ delegating decision rights to $\mathbf{A}$. A significant literature has since developed examining the merits of delegating authority. An assumption implicit across this entire literature (which we shall discuss presently) is that the sender is an expert at everything that is payoff relevant 1 We instead consider a scenario where the information relevant for $\mathbf{P}$ 's optimal decision is partly with $\mathbf{A}$ but the rest of it, that we call residual uncertainty, will become known at a later stage when the decision is to be taken. This paper presents a complete analysis of strategic communication in the presence of residual uncertainty and uses it to offer new insights on the delegation vs. authority debate.

A rough intuition for the costs and benefits of delegation and authority is as follows. The residual uncertainty causes the bias between the ex-post optimal action of $\mathbf{P}$ and A to be random. Giving the expert control over decisions (rather than just solicit her recommendation), and such a choice must be made in advance, would mean the principal exposes himself to risks of residual uncertainty. The extent of the loss depends on both the mean and the variance of the bias. If either of these is sufficiently large, $\mathbf{P}$ would naturally wish to retain authority since, by retaining control $\mathbf{P}$ avoids this risk, although he exposes himself to potential loss of the information held by $\mathbf{A}$ due to strategic reporting. Surprisingly, this loss of information could also be mitigated for the very fact that $\mathbf{P}$ 's decision will be random, from A's viewpoint, due to the residual uncertainty. Basically when contemplating misreporting her information, A weighs the possibility of triggering a decision close to her ideal position, if $\mathbf{P}$ 's bias happens to be congenial, against the possibility that the decision could go very wrong with $\mathbf{P}$ 's bias turning out in a different direction. The usual concavity of the payoff function now creates an incentive for $\mathbf{A}$ to make her information more precise to reduce the likelihood of these extreme decisions. As we shall see, the tradeoffs could be such that even truthful revelation becomes a possibility. Even when full revelation does not occur, the extent of information transmission increases rapidly enough with a fall in the mean bias so that keeping authority is optimal

\footnotetext{
1 Wolinsky (2002) is an exception. We discuss this in Section 4
} 
for $\mathbf{P}$.

Section 2 contains a formal description of the game of Strategic Information Transmission Under Uncertainty (hereafter, the SITU game). Essentially, the canonical payoffs of the CS model used in the literature are augmented to allow for additional sources of uncertainty apart from the type uncertainty regarding the sender. It turns out that the impact of the extraneous (or residual) uncertainty on the quality of equilibrium information transmission can be captured by a single parameter that we call the effective bias. We characterize all the equilibria of the SITU game with virtually no assumptions on the distribution of the residual uncertainty. Proposition 1 establishes that a fully revealing equilibrium exists if and only if the effective bias is zero. Proposition 2 characterizes equilibria when effective bias is non-zero. In particular it is shown that the equilibria are isomorphic to a certain Crawford and Sobel's information transmission game (which does not have any residual uncertainty). This in turn allows us to construct several comparative statics of the equilibria of the SITU game with respect to the mean and variance of residual uncertainty (see Proposition 3 and Proposition 4).

The comparative statics that we establish allow us to study the costs and benefits of delegation in a SITU game, just as in Dessein (2002) did for the CS-game. Section 3 contains this entire discussion. Through Proposition 5- Proposition 7, we show how the costs and benefits of delegation vary in relation to the mean and variance of the residual uncertainty and that of type uncertainty. In fact, Proposition 7 is a fairly general result which shows that for arbitrarily small variance in the residual uncertainty, $\mathbf{P}$ 's optimal decision to delegate is non-monotonic in the mean bias in the ex-post optimal actions of the two players: for small values and large values of the mean, $\mathbf{P}$ prefers to retain authority. This is in contrast with Dessein (2002)'s conclusion (effectively the case of zero variance of residual uncertainty) that $\mathbf{P}$ should prefer delegation in all such equilibria..$^{2}$

Proposition 4, an important ingredient to our discussion of the merits of delegation, may be of independent interest. Recall from Crawford and Sobel (1982) that the magnitude of a parameter $b$ determines the proximity of players' preferences and bounds the amount of information that is conveyed. A key question, unanswered in Crawford and Sobel (1982) or elsewhere, that we need to resolve is whether P's payoff in a CS-game converges to the fully revealing payoff as $\mathbf{b}$ converges to zero (and preferences of $\mathbf{P}$ and A coincide). While it is tempting to conjecture that it does, a formal proof of this fact is actually quite non-trivial. Proposition 4 answers this in the affirmative when a player's payoff in any state depends only on the distance from an optimal action. ${ }^{3}$

\footnotetext{
${ }^{2}$ Strictly speaking this does not negate Dessein's observation as our discussion is with regard to changing of residual uncertainty whereas his discussion is with regard to proximate preferences - the two trivially coincide when there is no residual uncertainty. This is discussed further in Section 3.

${ }^{3}$ The question of convergence to the fully revealing equilibrium payoff as the preferences of the two players tend to coincide has also been addressed by Spector (2000). Unfortunately, preferences in his
} 


\section{Literature Review}

\section{Strategic Information Transmission}

The literature has seen several extensions of the CS-game. By introducing additional elements to the basic CS-model such as multi-dimensional type uncertainty, partial verifiability of actions, multiple experts reporting on the state etc., the broad objectives have been how to improve the extent of information transmission in equilibrium, including even achieve full revelation of information (Gilligan and Krehbiel (1989), Seidmann and Winter (1997), Krishna and Morgan (2001), Krishna and Morgan (2004), Battaglini (2002), Levy and Razin (2007), Ambrus and Takahashi (2008), Li and Madarsz (2008), Chakraborty and Harbaugh (2010) etc.) 4 A common feature of all such extensions is that the expert or experts are fully aware of the state of the world (but the multiple experts may differ in their preferences). Our work differs from all these papers in that an expert knows only part of the payoff relevant information. This feature allows for possibly full revelation in a pure communication game with a compact state space and a single expert.

Seidmann (1990), Watson (1996), McGee (2009), Chen (2009) and de Barreda (2011) all consider the possibility that $\mathbf{P}$ observes additional signals. Except in Seidmann (1990), these signals do not directly affect the vNM utility of $\mathbf{P}$, rather they are useful due to their correlation with A's type. A SITU game is distinguished from these as we make the opposite assumption - the residual uncertainty is distributed independently of type uncertainty but affects the vNM utilities. In Section 4 we comment on how one may address some of the issues raised in those papers with our framework. In Seidmann (1990)'s model of communication, the sender is privately informed of one random variable whereas the receiver is privately informed of another random variable, correlated with the first. His analysis consists of three examples that violate some assumption of CS to suggest that "there can be effective cheap talk even in the least promising circumstances". In the first two examples he removes the single crossing property assumed in CS and introduces a condition labeled as (IA) under which all types of the sender agree on the relative ranking of any pair of possible decisions to be taken by the receiver. (This is also the case in Watson (1996).) In his Example 3, Seidmann considers non-scalar actions and the receiver's type is in fact common-knowledge. These are considerably different scenarios to the one considered in this paper. In summary, none of Seidmann's analysis

setting differ from those typically used in applications of the CS-game and cannot be used here. The reader will notice that the somewhat involved proof does seem not readily generalizable to more general payoff functions and a proof for the most general payoff functions considered by Crawford and Sobel (1982) remains open.

${ }^{4}$ Kartik et al. (2007) (also see Kartik (2009)) modify the CS-game so that messages inherently affect the payoffs, but now it is no longer a pure communication game. They show that if the state space is unbounded, there is a fully revealing signaling equilibrium. 
can therefore be ported to our study (or vice versa).

The SITU game studied here is however sufficiently general to embed some of the existing models in the literature. For instance, Harris and Raviv (2005) study the interaction between a CEO and a divisional manager with differing information about the global and local variables that affect investment decisions. Interestingly, the different states of uncertainty may also be reinterpreted as $\mathbf{P}$ really being a composition of multiple audiences. Accordingly, the case of public communication to multiple audiences explored in Goltsman and Pavlov (2011) can be regarded as essentially a SITU game. In fact we use these analogies to illustrate some of the aspects of our model in Example 1 and Example 2.

\section{On Delegation vs. Control}

There is a strand of the literature that discusses the merits of delegation in two-agent settings where the decision variable is contractible. These include Holmstrom (1984), Armstrong (1994), Melumad and Shibano (1991), Alonso and Matouschek (2007), Alonso and Matouschek (2008), Armstrong and Vickers (2010), among others. Similarly, Koessler and Martimort (2010) provide a screening model (with full commitment) where decision variables are multi-dimensional. The principal, with an upward bias in each dimension relative to the agent's ideal actions, optimally distorts the actions in opposite directions to create countervailing incentives to minimize his loss due to information asymmetry. While in contrast the decision variables in our setup are non-contractible, the basic intuition for communication in the two models is the same: translated in our case, different dimensions (of Koessler and Martimort) come from different realizations of the interim uncertainty. Unlike in the above literature, in the present model, the decision maker cannot commit ex-ante to any of the actions.

The key papers concerning delegation and communication when the decision variable is not contractible include Aghion and Tirole (1997), Ottaviani (2000), Dessein (2002), and Krahmer (2006). In all of these models the agent with the superior information is fully informed but may have possibly multi-dimensional information. In our model here, the type uncertainty of the expert is still one-dimensional, just as in Dessein (2002). By varying the mean and variance of the residual uncertainty faced by our partially informed agent, we are able to offer a wider perspective to the results in Dessein (2002) in particular. We show how even an arbitrarily small amount of residual uncertainty is often enough to ensure the superiority of control over delegation. An elaborate discussion of these issues is deferred to Section 3. 


\section{Strategic Information Transmission Under Uncertainty}

Recall the Crawford-Sobel game of strategic information transmission, hereafter the CSgame: there are two players, $\mathbf{P}$ and $\mathbf{A}$. The authority for choosing an action rests with $\mathbf{P}$, although the choice affects the payoffs of both players. Their payoffs also depend on the realization of a real-valued random variable, denoted by $\theta$, that is distributed with a density $f$ that is continuous and positive on a (non-degenerate) interval support $\Theta=\left[\theta_{\ell}, \theta_{h}\right]$. The game unfolds with $\mathbf{A}$ privately observing $\theta$ and then recommending an action to $\mathbf{P}$. $\mathbf{P}$ observes $\mathbf{A}$ 's recommendation and chooses an action $\xi \in \mathbb{R}$ and the game ends resulting in respective vNM payoffs of $\mathrm{U}_{\mathfrak{p}}(\xi, \theta)$ and $\mathrm{U}_{\mathfrak{a}}(\xi, \theta)$ to the two players. CS impose the fairly minimal conditions on the payoffs among which are the assumptions that for $i=a, p, U_{i}(\cdot, \theta)$ is differentiable and admits a unique maximum. Furthermore, $\mathrm{U}_{i 12}(\xi, \theta)>0$, a sorting condition, is also imposed which ensures that the ex-post optimal actions are monotonic in $\theta$.

In subsequent applications of the CS model in the literature, it is common to specify that $\mathrm{U}_{\mathrm{p}}(\xi, \theta)=-\ell_{\mathrm{p}}(|\xi-\theta|)$ and $\mathrm{U}_{\mathrm{a}}(\xi, \theta)=-\ell_{\mathrm{a}}(|\xi-\theta-\mathrm{b}|)$ respectively where $\ell_{i}$, for $i=a, p$, is a symmetric loss function that achieves a unique minimum at zero. Here $\mathbf{b} \neq 0$ is a constant. As the ex-post optimal actions of $\mathbf{P}$ and $\mathbf{A}$ differ by $\mathbf{b}$ in every state, we shall refer to this version of the CS-game as a CS-game with constant bias.

The SITU allows for the possibility that other sources of uncertainty in addition to the private information of A affect the players' payoffs. Accordingly, a true state of the world is now a tuple $(\theta, \mathbf{s})$ where $\mathbf{s}$ denotes the realization of a (possibly multivariate) random variable. Assume that $\theta$ is distributed independently of $\mathbf{s}$ according to density $\mathbf{f}$ as before. Assume also that $\mathbf{s}$ is drawn from an arbitrary (measurable) set $\mathcal{S} \subseteq \mathbb{R}^{k}$ according to a probability distribution function $\mathbf{G}$. We will refer to the uncertainty regarding $\mathbf{s}$ as residual uncertainty and $\theta$ as type uncertainty.

The rules of play for the SITU game are similar to those of the CS-game: A privately observes her "type" $\theta \in \Theta$ and sends $\mathbf{P}$ a message $\mathbf{m}$, chosen from a given message space $\mathcal{M}$. $\mathbf{P}$ observes A's report and the realization of residual uncertainty $\mathbf{s}$ and then chooses an action, which ends the game 5

The payoffs in the SITU game are a natural generalization of a CS game with constant bias. Given a pair of vectors $\mathbf{w}=\left(w_{1}, \ldots, w_{k}\right)$ and $\mathbf{s}=\left(s_{1}, \ldots, s_{k}\right)$, let $\mathbf{w} \cdot \mathbf{s}=\sum_{i=1}^{k} w_{i} s_{i}$.

\footnotetext{
${ }^{5}$ It will be clear that in terms of the timing of resolution of uncertainty, the determining feature is that $\mathbf{A}$ is unaware of the realization $\mathbf{S}$ at the communication stage and that $\mathbf{P}$ is fully aware of $\mathbf{s}$ prior to taking his action. Notice $\mathbf{A}$ acts only once - at the communication stage. Therefore, whether $\mathbf{A}$ eventually learns of the realization of $\mathbf{s}$ is really a moot point insofar as $\mathbf{A}$ 's incentives for information transmission are concerned. That is, we can allow $\mathbf{s}$ to either be privately observed by $\mathbf{P}$ or be publicly observable. (In Section 3 it will be necessary to specify if it is in fact publicly observed in order to compare the costs and benefits of delegation.)
} 
The vNM utility of $\mathbf{P}$ and $\mathbf{A}$ from an action $\xi \in \mathbb{R}$ in state $(\theta, \mathbf{s})$ are respectively

$$
\begin{aligned}
& u_{p}(\xi, \theta, s)=-\ell_{p}\left(\left|\xi-\theta-w_{p} \cdot s\right|\right), \\
& u_{a}(\xi, \theta, s)=-\ell_{a}\left(\left|\xi-\theta-w_{a} \cdot s\right|\right)
\end{aligned}
$$

where $\boldsymbol{w}_{\mathrm{a}}, \boldsymbol{w}_{\mathrm{p}} \in \mathbb{R}^{\mathrm{k}}$ are given vectors of coefficients that are common knowledge. Assume throughout that $\ell_{p}($.$) and \ell_{a}($.$) are both increasing, twice continuously differentiable$ convex functions. To keep the analysis interesting, assume that $\boldsymbol{w}_{\mathrm{a}} \neq \boldsymbol{w}_{\mathrm{p}}$, which keeps the preferences of the two players different.

To see that the above is a generalization of a CS-game with constant bias, take $\mathbf{s}$ to be real-valued and degenerate at some constant $b$, set $w_{p}=0, w_{a}=1$ and we have $u_{p}(\xi)=-\ell_{p}(|\xi-\theta|)$ and $u_{a}(\xi, b)=-\ell_{a}(|\xi-\theta-b|)$. The above specification of payoffs is also general enough to embed some of the other models studied in the literature.

Example 1 Let us begin by describing Harris and Raviv (2005), which studies the interaction between a CEO $(\mathbf{P})$ and a divisional manager $(\mathbf{A})$ in a company, as a SITU game. The profit maximizing investment depends on both a global parameter, say $\mathrm{g}$, privately observed by $\mathbf{P}$, and a local knowledge parameter, say $\theta$, privately observed by A. They specify the vNM loss from an investment $\xi$ to be of the form $\ell_{p}(|\xi-\theta-g|)$ and $\ell_{a}(|\xi-\theta-g-b|)$ where $b$ is a fixed constant. Observe that if $\theta$ and $g$ are known, the optimal investment of $\mathbf{P}$ is $\mathbf{y}=\theta+\mathbf{g}$, whereas the divisional manager, perhaps given her proclivity to empire building, wishes to invest $y+b$ (in this case presumably $b>0$ ). The game they study is one in which $\mathbf{A}$ (strategically) communicates her type and $\mathbf{P}$ chooses how much to invest. This is clearly a SITU game. In terms of our notation, take $\mathbf{s}=(\mathrm{g}, \mathrm{b})$ to denote the residual uncertainty and let $\mathbf{w}_{\mathrm{a}}=(1,1)$ and $\mathbf{w}_{\mathrm{p}}=(1,0)$. The distribution function $G$ can be chosen appropriately while recognizing that $b$ is $a$ constant.

Example 2 (Partnerships and Full Revelation) Next suppose we take $\mathbf{w}_{\mathrm{a}}=$ $(0,0)$ in Example 1 so now $\mathbf{A}$ 's loss function is $\ell_{\mathrm{a}}(|\xi-\theta|)$ and we let $\mathrm{g}$ take values $\mathrm{g}_{1}<\mathrm{g}_{2}$ with probability $p$ and $(1-p)$. Continuing on the above theme, we may interpret this as a scenario where the divisional manager only cares about the local variable and does not care about the global variables (nor has an incentive for empire building). Alternatively, one may follow the lead in Goltsman and Pavlov (2011) and think of $\mathbf{P}$ as a "partnership" of two members say $\mathbf{P} 1$ and $\mathbf{P} 2$. There is a probability $\mathbf{p}$ that the partner $\mathbf{P} 1$ takes actual policy decision and $\mathbf{P} 2$ takes the decision with the remaining probability. $\mathbf{A}$ is a lobbyist who privately observes $\theta$ and only cares about the distance of the ultimate choice from $\theta$.

Observe that if $\mathbf{P i}$ were to choose the action upon knowing the value of $\theta$, he would 
pick the action $y_{i}=\theta+g_{i}$ if $\theta$ is realized. Since $\mathbf{A}$ on the other hand wishes to choose $\theta$, the bias in their ex-post optimal actions is $g_{i}$, which is random. Interestingly, the uncertain bias, depending on the nature of its distribution, allows for the possibility of fully revealing equilibrium in the SITU game. To illustrate, suppose $-g_{1}=g_{2}=s_{0}>0$ and that they occur with equal probability, i.e. $p=1 / 2$. Assume that $\mathbf{A}$ 's loss function when of type $\theta$ is the symmetric U-shaped curve shown in the diagram below, which has a minimum at $\theta$.

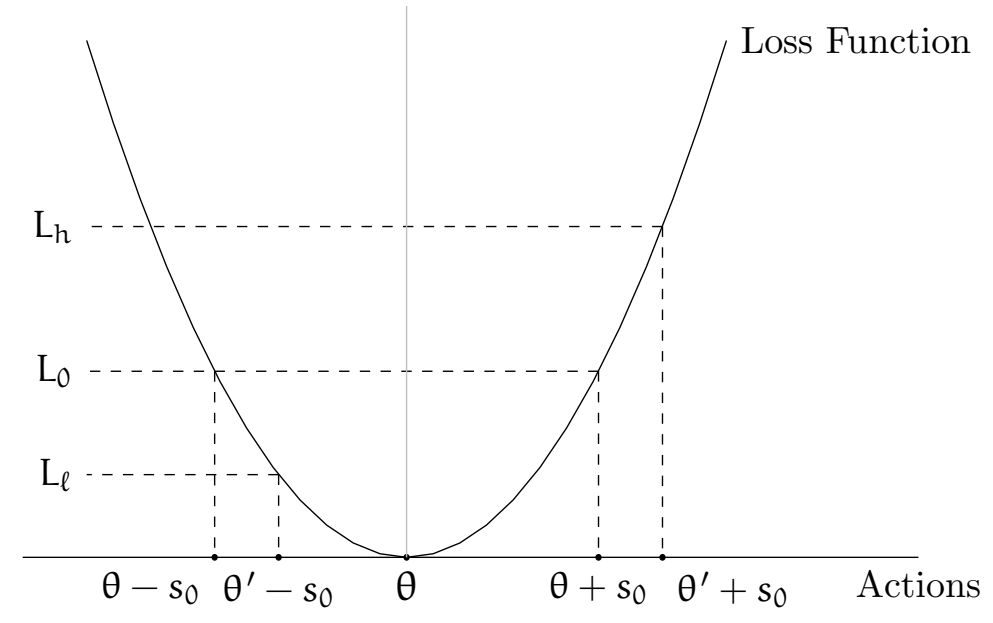

Figure 1: Full revelation in a SITU game

Consider A reporting her information truthfully to the partnership as a candidate equilibrium strategy. What are the incentives for $\mathbf{A}$ of type $\theta$ to report her type truthfully? Should $\mathbf{P} 1$ (or $\mathbf{P} 2$ ) be the decision maker, on hearing the report $\theta$, he would choose the action $y_{1}=\theta-s_{0}$ ( or $\left.y_{2}=\theta+s_{0}\right)$. From $A^{\prime}$ 's viewpoint, $y_{1}$ and $y_{2}$ are each selected with equal probabilities. This leads to an expected loss of $\mathrm{L}_{0}$. Now if $\mathbf{A}$ of type $\theta$ were to mimic $\theta^{\prime}>\theta$, the equally likely actions are $y_{1}^{\prime}=\theta^{\prime}-s_{0}$ and $y_{2}^{\prime}=\theta^{\prime}+s_{0}$, leading to a respective ex-post loss $\mathrm{L}_{\ell}$ and $\mathrm{L}_{h}$. Observe that the gain from that misrepresentation is $\left(\mathrm{L}_{0}-\mathrm{L}_{\ell}\right)$ when $-\mathrm{s}_{0}$ occurs, but this is not enough to offset the increased cost $\left(\mathrm{L}_{h}-\mathrm{L}_{0}\right)$ when $s_{0}$ occurs, due to the convexity of the loss function, causing a positive net expected loss. Following such logic, it is clear that such defections (and others) are dominated from which we can conclude that full revelation can be supported as an equilibrium behavior ${ }^{6}$ Later, Proposition 1 gives a necessary and sufficient condition for the existence of a fully revealing equilibrium.

\footnotetext{
${ }^{6}$ In the discussion following their Proposition 2 concerning public communication to multiple audiences, Goltsman and Pavlov (2011) in fact recognize this possibility of full revelation in the special case where there are two audiences. They also remark on the lack of an obvious generalization. The idea of an effective bias to be introduced shortly and Proposition 1 is precisely the required generalization.
} 
Example 3 (Uncertain vs. Privately Informed Principal) Prior to embarking on the more elaborate formal analysis, it is useful to illustrate the sharp contrast in the predictions of the SITU game to those obtained by assuming that both players are uncertain of the residual uncertainty. Assume in Example 1 that $b=0$ so that the divisional manager is not subject to empire building motives. When $(\theta, g)$ is commonknowledge, $\mathbf{P}$ and $\mathbf{A}$ share a common objective of investing $\theta+\mathbf{g}$. Therefore, in the SITU game, it is evident that there would be a fully revealing equilibrium. In contrast, if both players are uncertain of $\mathrm{g}$, and $\theta$ were common-knowledge, $\mathbf{P}$ 's most preferred action would be $\theta+\beta_{p}$ whereas A's most preferred action would be $\theta+\beta_{a}$ where, for $i=a, p$

$$
\beta_{i}=\arg \min _{\xi} E_{g}\left[\ell_{i}(|\xi-g|)\right]
$$

Observe that unless $\ell_{p}=\ell_{a}$, i.e. both players share the same loss function or some such restrictive assumption, typically $\beta_{p} \neq \beta_{a}$. The consequent bias in the ex-post optimal actions of the two players would prevent the existence of a fully revealing equilibrium, just as in the original CS game. Interestingly, the converse may also hold (see the discussion on "Correlation with Type Uncertainty" in Section 4.

\subsection{Ex-ante, Ex-post and Effective Bias}

Before we formally describe the strategies (and the equilibrium) in a SITU game, we introduce a few key concepts that are necessary for presenting our results. Observe that the ex-post optimal choices of $\mathbf{P}$ and $\mathbf{A}$ are respectively

$$
\begin{aligned}
& y_{p}(\theta, \mathbf{s})=\theta+\boldsymbol{w}_{p} \cdot \mathbf{s}, \\
& y_{a}(\theta, \mathbf{s})=\theta+\boldsymbol{w}_{a} \cdot \mathbf{s} .
\end{aligned}
$$

We will refer to

$$
b_{\mathrm{s}}:=\left(w_{\mathrm{a}}-w_{\mathrm{p}}\right) \cdot \mathrm{s},
$$

the difference between the ex-post optimal action of $\mathbf{A}$ and that of $\mathbf{P}$ as the ex-post bias. As indicated previously, if $\mathrm{G}$ is degenerate at some $\mathbf{s}$, then the SITU game is effectively a CS-game with the fixed bias $b_{\mathbf{s}}$. Of course, our concern in this paper is primarily in the case where $G$ is non-degenerate.

Let

$$
\mu_{\mathrm{b}}=\mathrm{E}\left[\mathrm{b}_{\mathrm{s}}\right] \text { and } \sigma_{\mathrm{b}}^{2}=\mathrm{E}\left[\mathrm{b}_{\mathrm{s}}-\mu_{\mathrm{b}}\right]^{2} \text {. }
$$


We will refer to $\mu_{\mathrm{b}}$ interchangeably as the ex-ante bias or the mean bias. $\sigma_{\mathrm{b}}^{2}$ is of course the variance of the ex-post bias.

With a non-degenerate $\mathrm{G}$, one may be tempted to guess that the mean bias acts as a proxy for determining the extent of information transmission in the SITU game. It turns out that due to strategic considerations, the relevant differences in players' preferences are instead captured by a statistic that in general differs from the mean bias. To define this, we first introduce the function $\varphi: \mathbb{R} \longrightarrow \mathbb{R}$ where

$$
\varphi(\xi)=\int \ell_{\mathrm{a}}\left(\left|\xi-\mathrm{b}_{\mathbf{s}}\right|\right) \mathrm{dG}(\mathbf{s})
$$

Note that $\varphi$ inherits the strict convexity of $\ell_{\mathrm{a}}(\cdot)$.

Definition 1 (Effective Bias) The effective bias in a SITU game is

$$
\mathrm{b}^{*}:=\operatorname{argmin}_{\xi} \varphi(\xi)
$$

(which is unique since $\left.\ell_{\mathrm{a}}^{\prime \prime}(\xi)>0\right)$.

The rationale for the above terminology is as follows: Fix the beliefs of $\mathbf{P}$ so that whenever he hears the report $\theta^{\prime}$ from $\mathbf{A}$, he believes it to be true. He would then take the action $\mathbf{y}_{p}\left(\theta^{\prime}, \mathbf{s}\right)$. The expected loss of $\mathbf{A}$ of type $\theta$ were to report her type to be $\theta^{\prime}$ is $\varphi(\eta-\theta)$. Therefore, a type $\theta$ would most prefer that $\mathbf{P}$ believes that she is of type $b^{*}+\theta .7$ Hence the term effective bias.

Remark 1 It will become clear in Proposition 1 and Proposition 2 that it is $\mathrm{b}^{*}$ that forms a "sufficient statistic" for determining the extent of information transmission that can occur in an equilibrium of the SITU game. It is worth emphasizing the distinction between effective bias and the mean bias. If for instance in Example 2, suppose the support of residual uncertainty is an arbitrary $g_{1}$ and $g_{2}$ with probability $p$ and and $(1-p)$ respectively, the effective bias and the mean bias are given by

$$
\mu_{\mathrm{b}}=p g_{1}+(1-p) g_{2} \text { and } p \ell_{\mathrm{a}}^{\prime}\left(\mathrm{b}^{*}-\mathrm{g}_{1}\right)=(1-\mathrm{p}) \ell_{\mathrm{a}}^{\prime}\left(\mathrm{g}_{2}-\mathrm{b}^{*}\right)
$$

respectively. Clearly, in general, $\mu_{\mathrm{b}}$ and $\mathrm{b}^{*}$ need not be equal and in particular, one can be zero without the other being zero. A point of natural interest is the coincidence of the mean bias and the effective bias. The following is a sufficient condition.

Condition A. Either (a) or (b) holds:

\footnotetext{
${ }^{7}$ This statement should be read with the caveat that a type $\theta$ whose type is such that $b^{*}+\theta \notin \Theta$ would prefer to mimic type $\theta_{\ell}$ if $b^{*}>0$ and $\theta_{h}$ if $b^{*}<0$.
} 
a. $\boldsymbol{A}$ 's loss function is quadratic, i.e. $\ell_{\mathrm{a}}(\xi)=\xi^{2}$.

b. The distribution of ex-post bias $\mathrm{b}_{\mathrm{s}}$ is symmetric.

Lemma 1 In any SITU game where Condition A holds,

1. $\varphi$ is symmetric around $\mu_{\mathrm{b}}$, i.e. $\varphi\left(\mu_{\mathrm{b}}+\xi\right)=\varphi\left(\mu_{\mathrm{b}}-\xi\right)$ for all $\xi \in \mathbb{R}$.

2. The effective bias is the same as the mean bias, i.e. $\mathrm{b}^{*}=\mu_{\mathrm{b}}$.

Proof. See Appendix.

\subsection{Equilibrium}

A strategy of $\mathbf{A}$ in the SITU game is any (measurable) function $\sigma_{a}: \Theta \longrightarrow \mathcal{M}$ and $\mathbf{P}$ 's strategy is a mapping $\sigma_{\mathrm{p}}: \mathcal{M} \times \mathcal{S} \longrightarrow \mathbb{R}$. Observe that, the composition of A's strategy with that of $\mathbf{P}$ in a given strategy profile yields an outcome function. That is, for any strategy profile $\left(\sigma_{a}, \sigma_{p}\right)$ consider the mapping $Y: \Theta \times \mathcal{S} \longrightarrow \mathbb{R}$ where

$$
Y(\theta, \mathbf{s})=\sigma_{\mathrm{p}}\left(\sigma_{\mathrm{a}}(\theta), \mathbf{s}\right)
$$

When the strategy profile $\left(\sigma_{a}, \sigma_{p}\right)$ is played, $Y(\theta, \mathbf{s})$ is the eventual action that will be chosen when the state is $(\theta, \mathbf{s})$. Using this, for an arbitrary strategy profile $\left(\sigma_{a}, \sigma_{p}\right)$, we can write down the expected loss of $\mathbf{A}$ when she is of type $\theta$ and chooses to deviate and mimic the behavior of type $\theta^{\prime}$ as:

$$
\mathrm{L}_{\mathrm{a}}\left(\theta^{\prime}, \theta\right)=\int \ell_{\mathrm{a}}\left(\left|\mathrm{Y}\left(\theta^{\prime}, \mathbf{s}\right)-\theta-\boldsymbol{w}_{\mathrm{a}} \cdot \mathbf{s}\right|\right) \mathrm{dG}(\mathbf{s}) .
$$

Remark 2 A SITU game is a multi-stage game of incomplete information with a continuum of types. Throughout, by an equilibrium, we mean a Perfect Bayesian Nash Equilibrium (see Fudenberg and Levine (1990)). In particular, we conduct the analysis using behavioral (pure) strategies whereas CS work with distributional strategies. This difference is inessential here.

Definition 2 (Equilibrium) An equilibrium consists of a strategy profile $\left(\sigma_{\mathfrak{a}}, \sigma_{\mathfrak{p}}\right)$ such that

$$
\mathrm{L}_{\mathrm{a}}\left(\theta^{\prime}, \theta\right) \geq \mathrm{L}_{\mathrm{a}}(\theta, \theta) \quad \forall \theta, \theta^{\prime} \in \Theta
$$


and for every $\mathrm{m} \in \mathrm{R}\left(\sigma_{\mathrm{a}}\right)$, where $\mathrm{R}\left(\sigma_{\mathrm{a}}\right) \subseteq \mathcal{M}$ denotes the range of $\sigma_{\mathrm{a}}$,

$$
\sigma_{p}(m, s) \in \operatorname{argmin}_{x} \int_{\theta^{\prime} \in \sigma_{a}^{-1}(m)} \ell_{p}\left(\left|x-\theta^{\prime}-w_{p} \cdot \mathbf{s}\right|\right) f\left(\theta^{\prime}\right) d \theta^{\prime},
$$

whenever $\sigma_{\mathrm{a}}^{-1}(\mathrm{~m})$ is of non-zero probability.

$\mathrm{Y}(\cdot, \cdot)$ is said to be an equilibrium outcome function $(E O F)$ of the SITU game if it is the outcome function of an equilibrium $\left(\sigma_{a}, \sigma_{p}\right)$.

Condition (5) is the usual incentive compatibility requirement on A's behavior. Condition (6) is the requirement that at every $m$ that is reported along the equilibrium path, $\mathbf{P}$ 's choice is a best response given his updated Bayesian posterior.

Equilibrium beliefs. Strictly speaking, the definition of an equilibrium must specify players' beliefs at all information sets, including out of the equilibrium path, as well as (5) and (6). Insofar as our concern is only in the characterization of the EOF, this is without loss of generality because, given a strategy profile $\left(\sigma_{a}, \sigma_{p}\right)$ such that (5) and (6) hold, pick $\hat{\theta}$ arbitrarily and let $\hat{\mathrm{m}}=\sigma_{\mathrm{a}}(\hat{\theta})$. For any $\mathrm{m} \in \mathcal{M} \backslash R\left(\sigma_{\mathrm{a}}\right)$, which represents an unreached node in the candidate equilibrium $\left(\sigma_{\mathfrak{a}}, \sigma_{\mathfrak{p}}\right)$, prescribe the beliefs of $\mathbf{P}$ at $\mathbf{m}$ to be the same as those at $\hat{\mathrm{m}}$ and redefine $\sigma_{\mathrm{p}}(\mathrm{m}, \mathbf{s})=\sigma_{\mathrm{p}}(\hat{\mathrm{m}}, \mathbf{s})$. That is, $\mathbf{P}$ behaves at any unreached equilibrium message exactly as he does upon hearing $\hat{\mathrm{m}}$. Since the original incentive compatibility conditions prevent any type (other than $\hat{\theta}$ ) from mimicking the behavior of $\hat{\theta}$, with the above prescribed beliefs, every type of $\mathbf{A}$ has an incentive to weakly report $\sigma_{\mathfrak{a}}(\theta)$ and makes $\left(\sigma_{\mathfrak{a}}, \sigma_{\mathfrak{p}}\right)$ a Perfect Bayesian Equilibrium, in the sense of Fudenberg and Levine (1990).

Observe that in any equilibrium $\mathbf{P}$ chooses an action only after observing $\mathbf{s}$. Since the ex-post optimal action is additively separable in $\theta$ and $\mathbf{s}$, it is intuitive that an EOF is similarly separable. The following Lemma makes this precise.

Lemma 2 For every EOF $\mathrm{Y}$ of the SITU game, there is a correspondingly unique function $\psi: \Theta \longrightarrow \mathbb{R}$ such that $Y(\theta, \mathbf{s})=\psi(\theta)+\boldsymbol{w}_{\mathrm{p}} \cdot \mathbf{s}$.

\section{Proof. See Appendix.}

The above characterization of EOF allows us to offer a simple necessary and sufficient condition for $\mathbf{A}$ to fully and truthfully reveal her information in equilibrium. Formally, an equilibrium $\left(\sigma_{a}, \sigma_{p}\right)$ is said to be fully revealing if its associated outcome function is $\mathrm{Y}(\boldsymbol{\theta}, \mathbf{s})=\theta+\boldsymbol{w}_{\mathrm{p}} \cdot \mathbf{s}$. A minimal necessary condition for the existence of fully revealing equilibrium is of course that the message space $\mathcal{M}$ is rich enough for each $\mathbf{A}$ type to distinguish itself in equilibrium. We shall say $\mathcal{M}$ is sufficiently rich provided there is an injective function from $\Theta$ to $\mathcal{M}$. An easy application of Lemma 2 combined with the 
definition of equilibrium yields the following complete characterization of existence of a fully revealing equilibrium.

\section{Proposition 1 (Fully Revealing Equilibrium) A fully revealing equilibrium} exists if and only if $\mathrm{b}^{*}=0$ and $\mathcal{M}$ is sufficiently rich.

Proof. Suppose a fully revealing equilibrium exists. The EOF is then $Y(\theta, \mathbf{s})=\theta+\boldsymbol{w}_{\mathrm{p}} \cdot \mathbf{s}$. Substituting in (4) we have

$$
\begin{aligned}
\mathrm{L}_{\mathrm{a}}\left(\theta^{\prime}, \theta\right) & =\int \ell_{\mathrm{a}}\left(\left|\theta^{\prime}+\boldsymbol{w}_{\mathrm{p}} \cdot \mathbf{s}-\theta-\boldsymbol{w}_{\mathrm{a}} \cdot \mathbf{s}\right|\right) \mathrm{dG}(\mathbf{s}) \\
& =\int \ell_{\mathrm{a}}\left(\left|\theta^{\prime}-\theta-\left(\boldsymbol{w}_{\mathrm{a}}-\boldsymbol{w}_{\mathrm{p}}\right) \cdot \mathbf{s}\right|\right) \mathrm{dG}(\mathbf{s}) \\
& =\varphi\left(\theta^{\prime}-\theta\right),
\end{aligned}
$$

and the equilibrium requirement (5) that $\mathrm{L}_{a}\left(\theta^{\prime}, \theta\right) \geq \mathrm{L}_{a}(\theta, \theta)=\varphi(0)$ gives $b^{*}=0$.

Conversely, assume $\mathrm{b}^{*}=0$. Since $\mathcal{M}$ is sufficiently rich, there is no loss of generality in assuming that $\mathcal{M} \supseteq \Theta$. Now suppose that $\mathbf{A}$ plays $\sigma_{a}(\theta)=\theta$ for all $\theta$. The equilibrium requirement (6) gives $\sigma_{p}(\theta, \mathbf{s})=\theta+\boldsymbol{w}_{p} \cdot \mathbf{s}$, which again gives $\mathrm{L}_{a}\left(\theta^{\prime}, \theta\right)=\varphi\left(\theta^{\prime}-\theta\right)$. Again, since $\mathrm{b}^{*}=0$, (5) holds as $\mathrm{L}_{\mathrm{a}}(\theta, \theta)=\varphi(0) \leq \varphi\left(\theta^{\prime}-\theta\right)=\mathrm{L}_{\mathrm{a}}\left(\theta^{\prime}, \theta\right)$ for all $\theta$.

Given the intuitive meaning of effective bias (following Definition 1), that this full revelation happens for $b^{*}=0$ in the SITU game is only natural. To see this recall that $\mathbf{b}^{*}$ is the extent by which a type would choose to distort her type if $\mathbf{P}$ were to believe A's report and choose optimally. That $b^{*}$ is generally different from the mean bias $\mu_{b}$ is crucial in understanding the difference between the SITU game and a CS-game of constant bias $b$, in general, and the full information revelation results in the two models, in particular. In the latter full revelation can occur when $b=0$ because $\mathbf{P}$ does exactly what $\mathbf{A}$ wants him to do. It is tempting to extend that idea to the SITU game and think that in the SITU game full revelation occurs because $\mathbf{P}$ does in expectation exactly what A wants him to do. Since $\mathbf{P}$ 's expected ex-post optimal action $E\left[y_{p}(\theta, s)\right]$ equals A's expected ex-post optimal action $\mathrm{E}\left[\mathrm{y}_{\mathrm{a}}(\theta, \mathbf{s})\right]$ only when $\mu_{\mathrm{b}}=0$, we would be led to the incorrect conclusion that full revelation occurs for $\mu_{\mathrm{b}}=0$. Indeed, as we discussed in Remark 1, $\mu_{\mathrm{b}}$ can vanish without $\mathrm{b}^{*}$ being zero (or vice versa), full revelation can occur without $\mathbf{P}$ doing in expectation what $\mathbf{A}$ would like him to do.

Full revelation of the expert's private information can occur in a SITU game even though this is a single sender-single receiver pure communication game, unlike say in Battaglini (2002) which has multiple senders or in Kartik et al. (2007) where messages affect the payoffs directly and signaling is a possibility 8 However, in such models full

\footnotetext{
${ }^{8}$ Improvement in information transmission can also occur due to errors in message transmission. (See for instance Myerson (1991)). Broadly speaking, errors in a game allow for a greater degree of correlated
} 
revelation is a generic possibility in the space of their parameters which is not the case here. Nonetheless, Condition A contains typical assumptions made in the literature and the fact that full-revelation would occur when this condition holds and $\mu_{\mathrm{b}}=0$, regardless of the variance of the ex-post bias, is of interest.

\subsection{Partition Equilibria}

When $b^{*} \neq 0$, full revelation is no longer possible. There are typically multiple (but finite) equilibria, each one distinguished by a partitioning of $\Theta$ into finitely many sub-intervals, just as in a CS-game. To characterize the equilibria, first define for any $a<a^{\prime}$,

$$
x\left(a, a^{\prime}\right)=\operatorname{argmin}_{\xi} \int_{a}^{a^{\prime}} \ell_{p}(|\xi-\theta|) f(\theta) d \theta
$$

to be the optimal action of $\mathbf{P}$ in the event he knows that $\theta$ lies in the interval $\left[\mathbf{a}, \mathbf{a}^{\prime}\right]$. Also let $\mathbf{a}=\left(a_{0}, a_{1}, \ldots, a_{n}\right)$ denote a typical partition of $\Theta$ into $\mathfrak{n}$ sub-intervals where $\theta_{\ell}=a_{0}<a_{1}<\cdots<a_{n}=\theta_{h}$ are the boundary points of the sub-intervals.

Proposition 2 Consider a SITU game with $\mathrm{b}^{*} \neq 0$.

1. In any equilibrium, there is a partition $\mathbf{a}=\left(\mathrm{a}_{0}, \mathrm{a}_{1}, \cdots, \mathrm{a}_{\mathrm{N}}\right)$ of $\Theta$ such that

$$
\varphi\left(x\left(a_{i-1}, a_{i}\right)-a_{i}\right)=\varphi\left(x\left(a_{i}, a_{i+1}\right)-a_{i}\right) \quad \forall i=1, \ldots, N-1,
$$

and the EOF is

$$
Y(\theta, \mathbf{s})=x\left(a_{i-1}, a_{i}\right)+w_{p} \cdot \mathbf{s}
$$

for all $\theta \in\left[\mathrm{a}_{\mathrm{i}-1}, \mathrm{a}_{\mathrm{i}}\right]$, for all $\mathrm{s} \in \mathcal{S}$ and for $\mathrm{i}=1, \ldots, \mathrm{N}$.

2. There exists a finite integer $\mathbf{N}^{*}$ such that an equilibrium described in Part 1 exists if and only if $\mathrm{N} \leq \mathrm{N}^{*}$.

Proof. See Appendix.

In words, just as in the CS-game, any equilibrium of the SITU game involves partitioning the state space $\Theta$ into some $\mathrm{N}$ sub-intervals. In what follows, we will refer to such an equilibrium as the $\mathrm{N}$-equilibrium and the corresponding partition as an equilibrium partition. All types of $\mathbf{A}$ within a sub-interval pool to send the same message. When $\mathbf{P}$

outcomes than are possible in an unmediated communication without, of course, changing the vNM payoffs of the underlying game. Blume et al. (2007) explores this phenomenon in the CS game with linear-quadratic payoffs. While payoffs are shown to improve, they also find that full revelation is an impossibility. 
knows that he is in the interval $\left(a_{i-1}, a_{i}\right)$, given his Bayes rational beliefs, his payoff from choosing an action $x+\mathbf{w}_{\mathrm{p}} \cdot \mathbf{s}$ is proportional to the minimand in the RHS of (7). Therefore, he chooses the action $x\left(a_{i-1}, a_{i}\right)$ with the correction $\boldsymbol{w}_{p} \cdot s$ for the residual uncertainty. The marginal type in each of the sub-intervals, $\theta=a_{i}$, is chosen so that she is indifferent between recommending $x_{i}=x\left(a_{i-1}, a_{i}\right)$ and recommending $x_{i+1}=x\left(a_{i}, a_{i+1}\right)$.

Remark 3 The fact that $\mathbf{P}$ 's choice occurs after observing $\mathbf{s}$ allows a natural isomorphism between the equilibria of a SITU game and a CS-game with the following utility specification:

$$
\begin{aligned}
& \mathrm{u}_{\mathrm{p}}(x, \theta)=-\ell_{\mathrm{p}}(|x-\theta|), \\
& \mathrm{u}_{\mathrm{a}}(x, \theta)=-\varphi(x-\theta) .
\end{aligned}
$$

To see this, we need only recall from Crawford and Sobel (1982) that a partition $\mathbf{a}=$ $\left(a_{1}, \ldots, a_{N}\right)$ satisfying the arbitrage conditions (8), together with the optimal choices of $\mathbf{P}$ given by $\chi\left(a_{i-1}, a_{i}\right)$ essentially characterize the equilibria of this CS-game. This remark generalizes the observation by Goltsman and Pavlov (2011) in their discussion of Part 2, Proposition 2 that we alluded to in Example 2. They mention how their multiple receiver model can be reinterpreted as the usual CS-game of constant bias in a certain special case. If we interpret each realization $\mathbf{s}$ as denoting a different receiver, the above discussion shows that a SITU game can be reinterpreted as a CS-game, although not necessarily one of constant bias. (Corollary 1 below explains when it can be interpreted as a CS-game with constant bias.)

Using the above relation between a CS-game and a SITU game, when additionally Condition A holds, by appealing to Lemma 1 and the implied symmetry of $\varphi$ around $\mu_{\mathrm{b}}$ we have the following:

Corollary 1 Suppose Condition A holds in a SITU game with a mean bias $\mu_{\mathrm{b}}$. Then the following hold:

1. $\mathbf{a}_{\mathrm{N}}=\left(\mathrm{a}_{0}, \mathrm{a}_{1}, \ldots, \mathrm{a}_{\mathrm{N}}\right)$ is an equilibrium partition of an $\mathrm{N}$-equilibrium of the SITU game if and only if

$$
a_{i}=\frac{\chi\left(a_{i-1}, a_{i}\right)+\chi\left(a_{i}, a_{i+1}\right)}{2}+\mu_{b} \quad \forall i=1, \ldots, N-1 .
$$

2. An $\mathrm{N}$-equilibrium is strictly Pareto-superior ${ }^{9}$ to an $\mathrm{N}-1$ equilibrium.

3. The equilibrium partition in Part 1 is identical to a CS-game of constant bias in which $\mathbf{P}$ 's loss function is $\ell_{\mathfrak{p}}$, and the bias is $\mu_{\mathrm{b}}$. In an $\mathbf{N}$-equilibrium of SITU game

\footnotetext{
${ }^{9}$ Throughout, assertions on Pareto comparisons are in terms of ex-ante payoffs.
} 
and the corresponding CS-game the payoff of $\mathbf{P}$ is given by:

$$
\pi_{N}^{p}\left(\mu_{b}\right)=-\sum_{i=1}^{n} \int_{a_{i-1}}^{a_{i}} \ell_{p}\left(\left|x\left(a_{i-1}, a_{i}\right)-\theta\right|\right) d F(\theta) .
$$

Proof of Corollary 1. Consider the CS-game of constant bias $\mu_{\mathrm{b}}$, the loss function $\ell_{\mathrm{p}}$ for $\mathbf{P}$ and any loss function for $\mathbf{A}$ that is symmetric about zero, for instance $\ell_{\mathrm{a}}$. The conditions listed as Eq. (9) and arbitrage conditions (A) in Crawford and Sobel (1982) that effectively characterize an equilibrium are precisely the arbitrage conditions (10), obtained by requiring a cutoff type $a_{i}$ to be indifferent between pooling with types in intervals $\left[a_{i-1}, a_{i}\right]$ and $\left[a_{i}, a_{i+1}\right]$. Moreover, by Lemma 1 and the implied symmetry of $\varphi$ around $\mu_{\mathrm{b}}$, (8) reduces to $10 \mathrm{n}$. Given this, the only part of the Corollary that requires proof is the claim of Pareto superiority of an $\mathrm{N}$-equilibrium payoff outcome relative to an $\mathrm{N}$-1-equilibrium payoff outcome.

To see this, note that a CS-game of constant bias satisfies the assumption (M), by Theorem 2 of CS. As a result, all their comparative static results apply. Furthermore, $\mathbf{P}$ 's payoff equals (11) both in the SITU game and the associated CS-game of constant bias. Therefore, the monotonicity of $\pi_{\mathrm{N}}\left(\mu_{\mathrm{b}}\right)$ with respect to $\mu_{\mathrm{b}}$ is assured. All that remains to be shown is that A's payoff in the SITU game also increases. But, a careful examination of the steps leading to Theorem 5 of CS reveals that the proof of that result can be carried forward ad verbatim after expecting out the residual uncertainty in A's payoff to conclude that $\mathbf{A}$ also prefers the equilibrium with a greater number of steps.

\subsection{Equilibrium Comparative Statics}

The extent of strategic information transmission clearly depends on the distribution of the residual uncertainty. It is of interest to see how the equilibrium properties change with respect to changes in residual uncertainty. Although it is possible to study this under slightly general conditions, to keep the analysis tractable, we shall assume that Condition A holds. Accordingly, for the rest of this Section then, fix $\ell_{p}, \ell_{a}$ and $f$ and assume that the residual $G$ varies in a way that Condition A holds so that Corollary 1 applies. Noting the isomorphism between an N-equilibrium of the SITU game with that of a CS-game of constant bias $\mu_{\mathrm{b}}$, many insights of Crawford and Sobel (1982) can now be brought to bear on our analysis. For instance, $\mathrm{N}^{*}$, noted in Part 2 of Proposition 2 equals the maximal number of elements in an equilibrium partition computed using CS's method for a CS-game of constant bias $\mu_{b}$; denote this by $\mathrm{N}_{\mu_{\mathrm{b}}}^{*}$.

Proposition 3 Consider a change in the distribution of residual uncertainty from $\mathrm{G}$ to $\mathrm{G}^{\prime}$ such that Condition $A$ applies. Let $\mu_{\mathrm{b}}$ and $\mu_{\mathrm{b}}^{\prime}$ be the corresponding mean bias. 
1. If $\mu_{\mathrm{b}}^{\prime}<\mu_{\mathrm{b}}$, then (a) $\mathrm{N}_{\mathrm{G}}^{*} \leq \mathrm{N}_{\mathrm{G}^{\prime}}^{*}$ and (b) The ex-ante payoff of $\mathbf{P}$ is higher in each corresponding $\mathrm{N}$-equilibrium, for all $\mathrm{N} \leq \mathrm{N}_{\mathrm{G}}^{*}$.

2. Suppose $\mu_{\mathrm{b}}^{\prime}=\mu_{\mathrm{b}}$ and the changed distribution of ex-post bias second order stochasti-

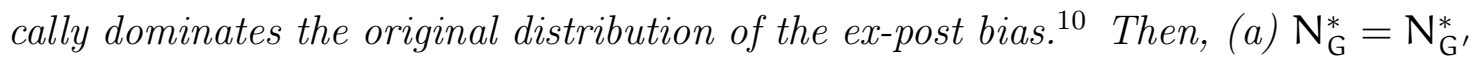
and (b) In the $\mathrm{N}$-equilibrium of $\mathrm{G}^{\prime}$, compared to the $\mathrm{N}$-equilibrium of $\mathrm{G}$, ex-ante, $\mathbf{P}$ is indifferent whereas $\mathbf{A}$ is better off.

Proof. See Appendix.

Part 1 of the proposition essentially follows from the insights of CS. An increase in the mean bias, via Corollary 1, simply means that the players are playing a CS-game with a higher constant bias. Part 1 is then little more than their well-known comparative statics. Of course, other parameters of residual uncertainty affect neither $\mathbf{N}_{\mu_{\mathrm{b}}}^{*}$ nor $\mathbf{P}$ 's payoff since $\mathbf{P}$ chooses his action after observing $\mathbf{s}$, and the arbitrage conditions 10 that determine the equilibrium partition depend only on $\mu_{\mathrm{b}}$ due to the symmetry of $\varphi$. It is worth noting, however, that in the CS game of constant bias A's payoff also increases with a fall in the bias. Such a result is not possible in the SITU game since the residual uncertainty affects A's payoff in a non-trivial manner. Part 2 therefore obtains only under the stronger condition of second order stochastic dominance.

Note that the above result only asserts that $\mathrm{N}_{\mu_{\mathrm{b}}}^{*}$ is non-decreasing in $\left|\mu_{\mathrm{b}}\right|$. However, for our later discussion on the merits of delegation, it is necessary to establish two facts, neither of which follow from the results in Crawford and Sobel (1982). First, that $\lim _{\left|\mu_{\mathrm{b}}\right| \rightarrow 0} \mathrm{~N}_{\mu_{\mathrm{b}}}^{*}=\infty$ and second, for any sequence of $\left\{\mathrm{N}^{k}\right\}$ such that $\mathrm{N}^{k} \leq \mathrm{N}_{\mu_{\mathrm{b}}}^{*}$, the size of each interval of the associated $\mathrm{N}^{\mathrm{k}}$-equilibrium partition converges to zero if $\mathrm{N}^{\mathrm{k}} \rightarrow \infty$. Together, these facts will imply that when $\left|\mu_{\mathrm{b}}\right|$ is small enough, the loss from strategic information transmission can be made close to zero in all sufficiently informative equilibria and consequently the payoff of $\mathbf{P}$ is arbitrarily close to his payoff under full revelation. Neither of the above facts follow directly from CS. We therefore prove the following Proposition that is crucial for later proving Proposition 7, a general result on the merits of delegation.

Recall that the mesh of a partition $\mathbf{a}=\left(a_{0}, a_{1}, \ldots, a_{N}\right)$ is the length of its longest sub-interval, denoted by $\|\mathbf{a}\|$.

Proposition 4 Fix $\ell_{\mathrm{p}}$ and consider a family of SITU games indexed by $\mu_{\mathrm{b}}$, all of which satisfy Condition A. Consider an infinite sequence $\left(\mu_{\mathrm{b}_{\mathrm{k}}}\right)$ such that $\lim _{\mathrm{k} \rightarrow \infty} \mu_{\mathrm{b}_{\mathrm{k}}}=0$. Then the following are true for the corresponding sequence of SITU games:

\footnotetext{
${ }^{10}$ Or equivalently, the original distribution of ex-post bias is a mean preserving spread of the changed distribution. To avoid mathematical technicalities that are costless with regard to economic intuition, the proof of this part is offered only for the case where the ex-post bias is bounded above.
} 
1. $\lim _{k \rightarrow \infty} \mathrm{N}_{\mu_{\mathrm{b}_{\mathrm{k}}}}^{*}=\infty$.

2. Consider any infinite sequence of integers $\left(\mathrm{N}_{\mathrm{k}}\right)$ such that $\mathrm{N}_{\mathrm{k}} \leq \mathrm{N}_{\mu_{\mathrm{b}_{\mathrm{k}}}^{*}}$ for all $\mathrm{k}$ and $\mathrm{N}_{k} \rightarrow \infty$. Then, for the corresponding equilibrium partitions $\mathbf{a}^{k}=\left(a_{0}^{k}, a_{1}^{k}, \ldots, a_{N_{k}}^{k}\right)$,

$$
\begin{array}{r}
\lim _{k \rightarrow \infty}\left\|\mathbf{a}_{k}\right\|=0, \\
\text { and } \lim _{k \rightarrow \infty} \pi_{N_{k}}\left(\mu_{b_{k}}\right)=-\ell_{p}(0) .
\end{array}
$$

Proof. See Appendix.

Since $\ell_{p}(0)$ is the payoff of $\mathbf{P}$ in a fully revealing equilibrium, Proposition 4 shows that the payoff of the most informative equilibrium at $\mu_{\mathrm{b}}$ is continuous at $\mu_{\mathrm{b}}=0$. The proof of Proposition 4 requires a novel argument that goes beyond Crawford-Sobel analysis and is somewhat involved. A sketch of the argument is as follows.

In an $\mathbf{N}$-equilibrium of a SITU game, before taking an action, $\mathbf{P}$ is only informed of the element of equilibrium partition $\mathbf{a}=\left(\mathrm{a}_{0}, \mathrm{a}_{1}, \ldots, \mathrm{a}_{\mathrm{N}}\right)$ that the realized $\theta$ lies in. This gives him a payoff of $\pi_{\mathrm{N}}\left(\mu_{\mathrm{b}}\right)$. Instead, suppose $\mathbf{P}$ could choose an arbitrary partition of $\Theta$ (into $N$ sub-intervals) and then take an action based on the knowledge of the interval that $\theta$ lies in. His most preferred partition, say $\mathbf{a}_{\mathrm{N}}^{*}=\left(\mathrm{a}_{0}^{*}, \mathrm{a}_{1}^{*}, \ldots, \mathrm{a}_{\mathrm{N}}^{*}\right)$, typically differs from $\mathbf{a}_{N}$ and leads to a payoff of say, $\pi_{N}^{*}$. What we show is that the difference between $\mathbf{a}_{N}^{*}$ and $\mathbf{a}_{N}$ is small when $\mu_{\mathrm{b}} \approx 0$ and consequently, $\pi_{N}\left(\mu_{\mathrm{b}}\right) \approx \pi_{\mathrm{N}}^{*}$. Separate arguments are then used to show that $\lim _{N \rightarrow \infty}\left\|\mathbf{a}_{N}^{*}\right\|=0$ (and hence $\lim _{N \rightarrow \infty} \pi_{N}^{*}=0$ ) and that $\mathrm{N}_{\mu_{\mathrm{b}}}^{*} \rightarrow \infty$ as $\mu_{\mathrm{b}} \rightarrow 0$ to complete the proof.

Given the association between a SITU game and a CS-game of constant bias, the above discussion may also be summarized by saying that $\mathbf{P}$ 's payoff in a CS-game of constant bias is close to zero if the bias is close to zero and one is looking at a sufficiently informative equilibrium. In this context, it is useful to compare Proposition 4 with the main result of Spector (2000). First, Spector considers payoffs in which the utility of an action $\xi$ is $\mathbf{U}(\xi)$ for $\mathbf{P}$ and $\mathbf{U}(\xi)+b V(\xi)$ for $\mathbf{A}$, where $\mathbf{U}$ and $\mathbf{V}$ are sufficiently well behaved functions. Ignoring different specifications of utilities, Proposition 4 is different from his main result in the following way. Spector's result is that for any $\left(b_{k}\right)$ such that $\lim _{k \rightarrow \infty} b_{k}=0$, there exists a sequence of $N_{k}$-equilibria along which the size of the largest interval in the corresponding equilibrium partition converges to zero. Clearly this also implies that $\lim _{k \rightarrow \infty} N_{k}=\infty$. Proposition 4, on the other hand, claims that the interval shrinks along every sequence of $N_{k}$-equilibria such that $\lim _{k \rightarrow \infty} N_{k}=\infty$. At a later stage when we discuss the merits of retaining authority over delegation, there is an equilibrium selection issue. The stronger claim of Proposition 4 goes some way in mitigating those considerations. 


\section{Delegation vs. Authority}

The complete characterization of the SITU game given in Proposition 1 and Proposition 2 enables us to evaluate the incentives of $\mathbf{P}$ to delegate authority to $\mathbf{A}$ instead of eliciting $\mathbf{A}$ 's information through communication. In our context, one may conduct the comparison along two different dimensions. One is to investigate the pros and cons of delegation with respect to variations in the residual uncertainty. The other possibility is to fix the residual uncertainty and investigate the incentive of $\mathbf{P}$ to retain authority, as preferences of $\mathbf{P}$ and $\mathbf{A}$ get closer - which is in the spirit of Dessein $(2002)$ - i.e. for $\left\|\mathbf{w}_{\mathfrak{p}}-\mathbf{w}_{\mathbf{a}}\right\| \approx 0$. Our focus for the most part is on the former case but we will return to the issue of proximate preferences later on in this section.

An a priori case for investigating the superiority of retaining authority from $\mathbf{P}$ 's viewpoint is presented by Proposition 1. When this proposition applies, which for instance is the case when Condition $\mathrm{A}$ holds and $\mu_{\mathrm{b}}=0$, there is after all a fully revealing equilibrium. If players were to coordinate on this equilibrium,

- It can be optimal for $\mathbf{P}$, even in an ex-post sense, to retain control, despite the fact that $\mathbf{A}$ is better informed than $\mathbf{P}$.

- If $\mathbf{P}$ could choose when to seek advice from $\mathbf{A}$, it is optimal for $\mathbf{P}$ to do so before the residual uncertainty is realized.

In fact, the above observations would be true even if we assumed that $\mathbf{s}$ is privately observed by $\mathbf{P}$. In the remainder of this section, we shall focus on the case where a fully revealing equilibrium does not exist.

Recall that typically a SITU game admits multiple equilibria. ${ }^{11}$ As P's payoff varies across these equilibria, the benefit of retaining authority involves an equilibrium selection problem. We shall therefore begin with providing a lower bound on the cost of delegation as this will enable us to answer the preliminary question of when authority can dominate delegation regardless of the equilibrium choice.

To evaluate the costs of delegation it is necessary to specify in greater detail A's information regarding the residual uncertainty at the time of making her choice, should $\mathbf{P}$ delegate authority. For most of the analysis, we shall examine the case where, if authority is delegated, A can choose an action after observing the realization of the residual uncertainty, just as $\mathbf{P}$ could in the SITU game. Toward the end we consider some variations in the timing of resolution of uncertainty and the delegation decision.

With the above assumption, it is clear that if $\mathbf{P}$ were to delegate authority, $\mathbf{A}$ takes the action $x_{a}(\theta, \mathbf{s})=\theta+\boldsymbol{w}_{\mathrm{a}} \cdot \mathbf{s}$ in state $(\theta, \mathbf{s})$. Hence, $\mathbf{P}$ 's payoff from delegation is

\footnotetext{
${ }^{11}$ Note that a fully revealing equilibrium is always accompanied by many non-revealing equilibria. Therefore, the issue of equilibrium selection remains even in this case.
} 
$-E\left[\ell_{p}\left(\left|b_{s}\right|\right)\right]$. Let $K:=\min _{\xi \in \Theta} \ell_{p}^{\prime \prime}(\xi) / 2$ Using Taylor's expansion of $\ell_{p}$ about $\mu_{b}$ with the Lagrange form for the remainder term, we have $\mathbf{P}$ 's payoff from delegation is bounded above by $\bar{\pi}_{\mathrm{D}}\left(\mu_{\mathrm{b}}, \sigma_{\mathrm{b}}^{2}\right)$, where

$$
\bar{\pi}_{\mathrm{D}}\left(\mu_{\mathrm{b}}, \sigma_{\mathrm{b}}^{2}\right)=-\ell_{\mathrm{p}}\left(\left|\mu_{\mathrm{b}}\right|\right)-K \sigma_{\mathrm{b}}^{2}
$$

If $\mathbf{P}$ keeps authority, since he takes an action after observing $\mathbf{s}$, he is completely protected from the variability in the residual uncertainty. On the other hand, once $\mathbf{P}$ delegates, he has no means of insuring against the variability of A's choice as it varies with the residual uncertainty, as is evident from the above bound on $\mathbf{P}$ 's payoff from delegation. By keeping authority however, $\mathbf{P}$ has to endure the loss of information regarding $\theta$. In the event the fully pooling equilibrium is played so that no information about $\theta$ is conveyed, $\mathbf{P}$ optimally chooses $\chi\left(\theta_{\ell}, \theta_{h}\right)$ resulting in a payoff of

$$
-\int_{\theta \in \Theta} \ell_{p}\left(\left|\theta-x\left(\theta_{\ell}, \theta_{h}\right)\right|\right) d F(\theta)
$$

which is of course independent of the residual uncertainty. Moreover, in any informative $\mathrm{N}$-equilibrium with $\mathbf{N}>1, \mathbf{P}$ is only better off (see Part 2, Corollary 1) relative to the babbling equilibrium payoff above. Comparing (12) with (13), we have the following simple and intuitive observation:

Proposition 5 In any SITU game, if either the mean or the variance of the ex-post bias is sufficiently large, $\mathbf{P}$ 's payoff is higher under authority, in every equilibrium of the SITU game than under delegation.

When Condition A holds, Corollary 1 tells us that $\mathbf{P}$ 's payoff in an $\mathrm{N}$-equilibrium of the SITU game is $\pi_{N}\left(\mu_{b}\right)$, the payoff that $\mathbf{P}$ would have received in an $\mathrm{N}$-equilibrium of a CS-game with a fixed bias $\mu_{\mathrm{b}}$. Notably, this payoff does not depend on $\sigma_{b}^{2}$. It is then immediate that, for any SITU game of a given mean bias $\mu_{b}, \mathbf{P}$ strictly prefers retaining authority provided the variability in the residual uncertainty, $\sigma_{b}^{2}$ is sufficiently large. Further, the payoff $\mathbf{P}$ would have received from delegation in the CS-game is $-\ell_{p}\left(\left|\mu_{b}\right|\right)$, which is less than the upper bound $\bar{\pi}_{D}\left(\mu_{b}, \sigma_{b}^{2}\right)$ on the analogous payoff in the SITU game. Therefore, all the conclusions of Dessein (2002) that establish the superiority of authority over delegation generalize naturally to our setup. This proposition is in this sense an analogue of his Proposition 4.

\footnotetext{
${ }^{12} \mathrm{~K}$ is well-defined since $\ell_{\mathrm{p}}$ is assumed to be twice continuously differentiable. Further, since we assumed that $\ell_{\mathrm{p}}$ is strictly convex, $\mathrm{K}>0$.
} 


\subsection{Small Variance of Ex-Post Bias and Delegation}

The sufficient condition provided in Proposition 5 should be expected. Indeed, $\mathbf{P}$, by retaining authority, is able to completely hedge against the residual uncertainty whereas under delegation, he is unable to do so. A higher mean and (being risk averse) a higher variance in the ex-post bias then increase the cost of delegation in predictable ways leading $\mathbf{P}$ to prefer authority.

Recall that depending on the size of effective bias, there may be several equilibria. A salient feature of Proposition 5 is that it is agnostic as to which equilibrium of the SITU game is played. However, since $\mathbf{P}$ 's payoff across $\mathrm{N}$-equilibria is increasing in $\mathrm{N}$, the minimal value of the variance of ex-post bias that is required for delegation to become a poor strategy, for a given $\mu_{\mathrm{b}}$ would be lower as we select a more informative equilibria. In fact it turns out that this minimal value of variance falls rapidly as $\mathrm{N}$ increases. To appreciate this, consider for the moment (we will consider more general preferences later) the SITU game where $\mathbf{P}$ has a quadratic loss function. By borrowing some of the calculations regarding the linear quadratic CS-game of a fixed bias in Section 4 of Crawford and Sobel (1982), we have the following proposition.

Proposition 6 Consider a SITU game in which $\ell_{\mathrm{p}}(\xi)=-\xi^{2}$, Condition A holds and $\theta$ is uniformly distributed over $[0,1] . \quad$ Fix any $\left(\mu_{\mathrm{b}}, \sigma_{\mathrm{b}}^{2}\right)$. For any $\mathrm{N} \leq \mathrm{N}_{\mu_{\mathrm{b}}}^{*}$,

1. $\mathbf{P}$ strictly prefers to retain authority in an $\mathbf{N}$-equilibrium game if and only if

$$
\sigma_{b}^{2}>\frac{1}{12 N^{2}}+\mu_{b}^{2}\left(\frac{\left(N^{2}-4\right)}{3}\right)
$$

2. $\mathbf{P}$ strictly prefers to retain authority in an $\mathbf{N}$-equilibrium whenever

$$
\sigma_{b}^{2}>\frac{\left(2 N^{2}+2 N-3\right)}{N^{2}(N+1)^{2}} \sigma_{\theta}^{2}
$$

Proof. See Appendix.

Part 2 of Proposition 6 offers a sufficient condition for dominance of authority by relating the variance in type uncertainty with the variance in the residual uncertainty. The actual magnitudes are interesting. For instance, if $\mathrm{N}=2$ then the ex-post bias need only be $25 \%$ more variable than type-uncertainty; with $\mathrm{N}=4$, ex-post bias needs to be only $10 \%$ more variable. In fact, the rate at which the ratio $\sigma_{b}^{2} / \sigma_{\theta}^{2}$ must fall is of the order of $\mathrm{O}\left(\mathrm{N}^{-2}\right)$. In other words, as the communication equilibrium becomes more informative, the incentive to retain authority becomes more attractive at a fairly rapid rate. Of course, one needs to bear in mind that in order to ensure the existence of an $\mathrm{N}$-equilibrium for $\mathrm{N}$ large enough, $\mu_{\mathrm{b}}$ must be sufficiently small. 


\section{Non-monotonicity of Delegation}

Part 1 of Proposition 6 offers a comparison that is based only on parameters of the residual uncertainty $\left(\mu_{\mathrm{b}}\right.$ and $\sigma_{\mathrm{b}}^{2}$ ). As such, it allows us to place the conclusions of Dessein (2002) in a wider context. For example, the curve $C$ in Figure 2 describes the pairs $\left(\mu_{b}, \sigma_{b}^{2}\right)$ such that (14) holds as an equality under the further assumption that the players coordinate on the most informative equilibrium $N_{\mu_{b}} \cdot 13$ Whenever $\left(\mu_{b}, \sigma_{b}^{2}\right)$ lies below the curve $\mathbf{C}$ (in the shaded area), delegation yields a higher payoff to $\mathbf{P}$ than authority while the opposite holds above C. Given Proposition 5, the interest is in what happens for smaller values of $\left(\mu_{b}, \sigma_{b}^{2}\right)$. When $\sigma_{b}^{2}=0$, which is precisely the case considered by Dessein $(2002)$, there

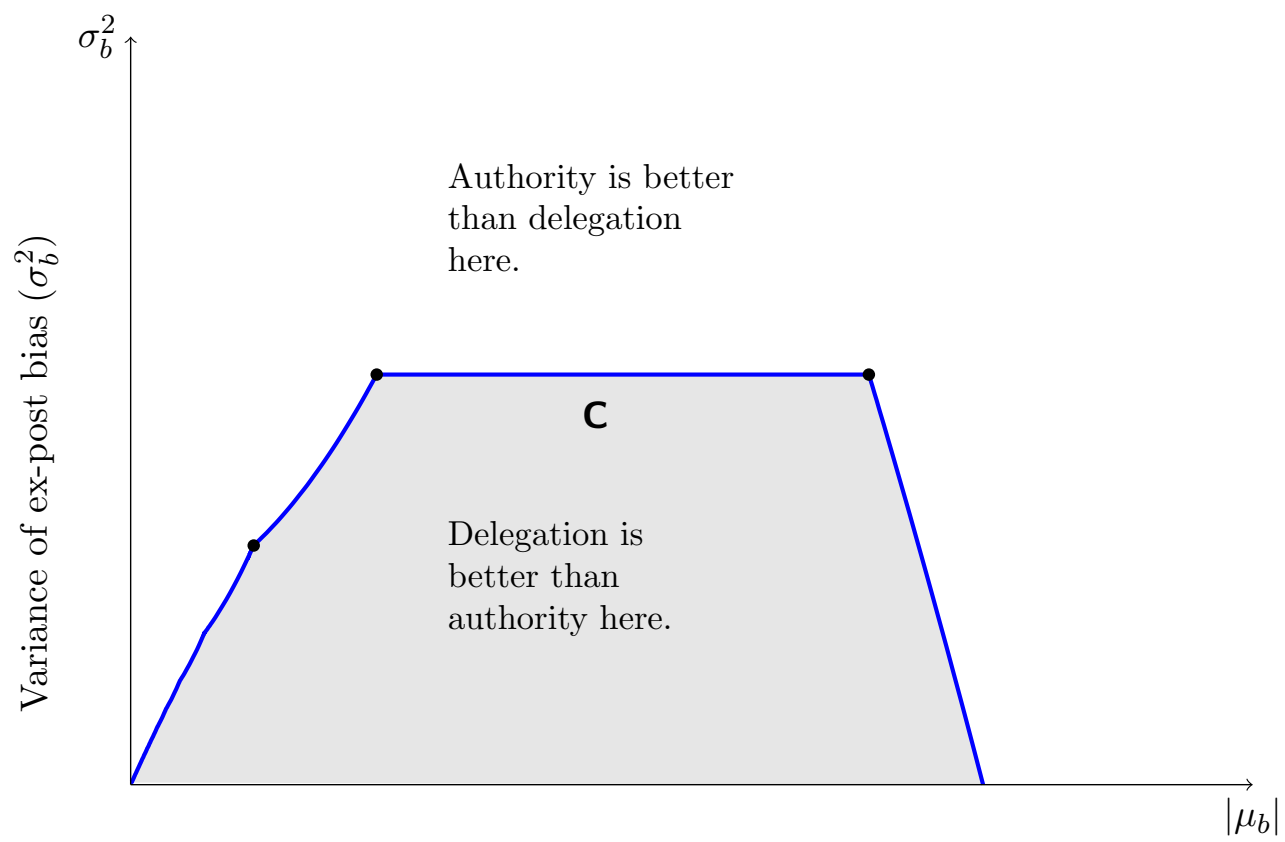

Mean of ex-post bias $\left(\mu_{b}\right)$

Figure 2: Delegation vs. Authority

is a threshold value of $\mu_{\mathrm{b}}$ below which delegation is superior - no matter how small the mean bias. As is evident in the picture, this is no longer true in the presence of some residual uncertainty. Indeed, for any positive $\sigma_{b}^{2}$, there is a threshold mean bias $\mu_{\mathrm{b}}$ below which we have the opposite conclusion: retaining authority is better for $\mathbf{P}$.

The non-monotonicity of the superiority of delegation/authority with respect to $\mu_{\mathrm{b}}$ for a fixed $\sigma_{b}^{2}>0$ is not a-priori obvious. For both the loss from delegation and the loss from authority are (weakly) decreasing in $\mu_{\mathrm{b}}$. In the context of the linear quadratic example, the loss from delegation is $\mu_{b}^{2}+\sigma_{b}^{2}$, and therefore increases at a unit rate with respect to $\mu_{\mathrm{b}}^{2}$. On the other hand, in a $\mathrm{N}$-equilibrium, the rate of increase with respect to $\mu_{b}^{2}$ is $\left(N^{2}-1\right) / 3$. For smaller values of $\mu_{b}^{2}$ that permit an equilibrium with $N \geq 3$

\footnotetext{
${ }^{13}$ See Chen et al. (2008) for a justification for selecting the most informative equilibrium.
} 
elements, a decrease in $\mu_{\mathrm{b}}^{2}$ is associated with a more than proportional decrease in the loss from keeping authority relative to delegation. Since for a $\left|\mu_{b}\right|$ close enough to zero, (a) the loss from delegation is bounded away from zero due to $\sigma_{b}^{2}$ being positive, while (b) the loss from authority converges to zero since there is enough transmission of information (i.e. $N_{\mu_{b}}^{*}$ is sufficiently large), authority dominates for a $\left|\mu_{b}\right|$ below a certain threshold.

Now, it is apparent from (12) that (a) holds generally and (b) too should be expected to hold because of Proposition 4. As a result, we are able to prove the following:

Proposition 7 Let $\ell_{\mathrm{p}}$ and $\epsilon>0$ be given. There exists an $\mathrm{N}_{\epsilon}$ such that for any SITU game (in which $\mathbf{P}$ 's loss function is $\ell_{\mathrm{p}}$ ) that satisfies Condition $A$, if $\sigma_{\mathrm{b}}^{2}>\epsilon$ and $\mu_{\mathrm{b}}$ is small enough so that an $\mathbf{N}$-equilibrium with $\mathbf{N} \geq \mathbf{N}_{\epsilon}$ exists, $\mathbf{P}$ prefers to retain authority in such an equilibrium.

Proof. See Appendix.

The upshot of Proposition 7 (along with Proposition 5) is that the non-monotonicity of superiority of authority over delegation with respect to the mean bias extends beyond the linear quadratic example to all loss functions, provided Condition A holds.

\subsection{Proximate Preferences and Delegation}

The above result (Proposition 7) should be interpreted carefully. It points out the "insurance value" of retaining authority which is absent when there is no residual uncertainty. That is, our model introduces an additional benefit of centralization that was absent in Dessein (2002). This, however, should not be seen as lessening one of Dessein's key observation which is that as the preferences of $\mathbf{P}$ and $\mathbf{A}$ become more aligned (i.e., bias disappears in the limit), the delegation turns out to be optimal. Recast in our setting, Dessein's result still holds for the following reason. While our comparative statics are performed in terms of the expected bias $\mu_{b}=E\left[b_{s}\right]$ and the variance $\sigma_{b}^{2}=E\left\{\left[b_{s}-\mu_{b}\right]^{2}\right\}$, the latter being the source of the insurance motive, the two are related. In particular, since the true ex-post bias is $\mathrm{b}=\left(\boldsymbol{w}_{\mathrm{a}}-\boldsymbol{w}_{\mathrm{p}}\right) \cdot \mathbf{s}$, therefore the mean bias $\mu_{\mathrm{b}}=\left(\boldsymbol{w}_{\mathrm{a}}-\boldsymbol{w}_{\mathrm{p}}\right) \cdot \mathrm{E}[\mathbf{s}]$ and $\sigma_{\mathrm{b}}^{2}=\left(\boldsymbol{w}_{\mathrm{a}}-\boldsymbol{w}_{\mathrm{p}}\right)^{\mathrm{T}} \cdot \boldsymbol{\Sigma}_{\mathrm{s}} \cdot\left(\boldsymbol{w}_{\mathrm{a}}-\boldsymbol{w}_{\mathrm{p}}\right)$, where $\boldsymbol{\Sigma}_{\mathrm{s}}$ is the variance-covariance matrix of $\mathbf{s}$. Now, talking about incentive alignment à $l a$ Dessein (2002) amounts to taking $\left\|\boldsymbol{w}_{\mathrm{a}}-\boldsymbol{w}_{\mathrm{p}}\right\| \rightarrow 0$ which has nothing to do with the shape of the underlying uncertainty itself. But as $\left\|\boldsymbol{w}_{\mathrm{a}}-\boldsymbol{w}_{\mathrm{p}}\right\| \rightarrow 0$, both $\mu_{\mathrm{b}}$ and $\sigma_{\mathrm{b}}^{2}$ become small. Further, $\sigma_{\mathrm{b}}^{2}$ converges to zero faster than $\mu_{\mathrm{b}}$ and the insurance motive disappears in alignment as well, confirming Dessein's observation. This, however, does not negate the basic point of our exercise which is that if we have increased residual uncertainty, e.g., increase the diagonal elements of $\Sigma_{\mathbf{s}}$ (keeping off-diagonal terms zeros, say), the cost of delegation increases because $\mathbf{A}$ would respond in a manner disliked by $\mathbf{P}$. 


\subsection{Timing of Communication and Uncertainty}

So far in our discussion of control vs. delegation, we have assumed that $\mathbf{P}$ elicits information from A before $\mathbf{S}$ is publicly observed. A natural question is whether $\mathbf{P}$, prior to the resolution of any uncertainty, has an incentive to commit to postpone the decision to either elicit information about $\theta$ or to delegate until after $\mathbf{s}$ is publicly observed. Proposition 7 offers a ready answer: if the mean bias is close to zero, $\mathbf{P}$ has no such incentives. For, if communication occurs after $\mathbf{s}$ is publicly observed, then it is as if $\mathbf{P}$ and $\mathbf{A}$ play the CS-game with a constant bias. On the other hand, if the residual uncertainty is such that $\left|\mathbf{b}_{\mathbf{s}}\right| \approx 0$ for all $\mathbf{s}$, we know from Dessein (2002) that $\mathbf{P}$ is better off by delegating authority giving him a loss of $\ell_{p}\left(\left|b_{s}\right|\right)$. Therefore, the expected loss from delaying either communication or delegation until after the realization of $\mathbf{s}$ leads to an expected loss for $\mathbf{P}$ that is bounded away from zero provided $\sigma_{b}^{2}>0$ and the support of $b_{\mathbf{s}}$ contains a set of positive measure close to 0 . However, if the mean bias is sufficiently close to zero, then eliciting information on $\theta$ prior to public revelation of $\mathbf{s}$ allows the information loss to be arbitrarily close to zero (see Proposition 4 and Proposition 7). In this case authority dominates delegation.

\section{Concluding Remarks}

In this section, we shall discuss a few future research possibilities.

\section{Interim Delegation}

We considered the question of delegation vs. control in a hierarchy with a sequential resolution of multiple sources of uncertainty. An introduction of an additional uncertainty arising in the post-delegation stage exposes the principal to new risks as the agent's ex-post optimal decisions might significantly differ from that of the principal. Even for small ex-ante differences between agent's preferences and that of the principal (as measured by the average bias of the agent), the principal likes to retain authority and tolerate loss of information due to agent's strategic communication, thus uncovering a completely different aspect of an important observation in the literature (Dessein (2002)) that the authority should be delegated to the agent. Given that the residual uncertainty considered in this paper is a very reasonable description, the lesson from our analysis is equally important.

A scenario that we have not covered is the case where $\mathbf{P}$ observes $\mathbf{S}$ privately and then decides whether to delegate or elicit information. We may call this interim delegation. In principle, the entire analysis of Section 2 can be used to study this case; $\mathbf{P}$ 's decision to retain authority provides information to A about the former's observation of $\mathbf{s}$. Given $\mathbf{P}$ 's equilibrium behavior, at any part of the game tree where he retains authority, there is 
a SITU game with A's posterior (which must be consistent with P's behavior in equilibrium) on $\mathbf{s}$ describing the nature of uncertainty. Our earlier analysis in this paper helps to characterize equilibrium behavior on that sub-tree. One may then work recursively to solve the entire game. We expect to pursue this in future work.

\section{Multiple Experts}

Throughout we have considered only a single agent. This is a reasonable assumption and even necessary to analyze the case with multiple experts. After all, in many organizational settings it is common to have a single expert, typically held on a retainer, and additional opinions are sought only on an ad hoc basis. Here, we shall briefly suggest how our analysis might be extended to multiple experts. A complete analysis would constitute a separate paper.

Indeed, suppose that there are $K$ agents and agent $i$ privately observes a signal $s_{i}$. Let $\mathbf{w}_{\mathrm{p}}=(1, \ldots, 1) \in \mathbb{R}^{\mathrm{K}}$ and for each $i=1, \ldots, \mathrm{K}$, let $\mathbf{W}$ be a $\mathrm{K} \times \mathrm{K}$ matrix such that every diagonal entry is ' 1 '. Let $\mathbf{w}_{\mathfrak{i}}$ denote the ith row of this matrix. Let $G$ denote the joint probability distribution of $\mathbf{s}$ on a compact support $\mathcal{S} \subset \mathbb{R}^{\mathrm{K}}$. Let $\ell_{\mathrm{p}}\left(\left|\boldsymbol{\xi}-\mathbf{w}_{\mathrm{p}} \cdot \mathbf{s}\right|\right)$ and $\ell_{i}\left(\left|\xi-\mathbf{w}_{i} \cdot \mathbf{s}\right|\right)$ denote the payoffs of $\mathbf{P}$ and agent $i$ when the true state is $\mathbf{s}=\left(s_{1}, \ldots, s_{K}\right)$. Assume that agents submit reports simultaneously.

The above informational setting is precisely the one found in Wolinsky (2002). However, the specification of preferences is different. In Wolinsky (2002) all the experts are biased in the same direction whereas here, two experts may be biased in different directions relative to $\mathbf{P}$ depending on the realization of the uncertainty. In terms of the SITU game, in the current setting, given the behavior of remaining agents, it is as if agent $i$ knows her type $s_{i}$ (which is $\theta$ in our earlier notation) but has a residual uncertainty about $\mathbf{s}_{-i}$. We can therefore proceed just as we did in Section 2.1 and define the ex-post bias of $i$ and the $\varphi_{i}$ function as follows:

$$
\begin{aligned}
b_{s}^{i} & =\left(\mathbf{w}_{i}-\mathbf{w}_{\mathfrak{p}}\right) \cdot \mathbf{s} \\
\varphi_{i}(\xi) & =\int_{\mathbf{s}_{-i} \in \mathcal{S}_{-i}} \ell_{i}\left(\left|\xi-b_{s_{-i}}\right|\right) d G_{-i}\left(\mathbf{s}_{-i}\right) .
\end{aligned}
$$

Finally, define the effective bias of agent $i$ as

$$
\mathrm{b}_{i}^{*}=\arg \min _{\xi} \varphi_{i}(\xi)
$$

Proceeding exactly as in the proof of Proposition 1 , we may now readily conclude that

Proposition 8 A necessary and sufficient condition for full revelation in the above game is that $\mathrm{b}_{\mathfrak{i}}^{*}=0$ for all $\mathrm{i}=1, \ldots, \mathrm{K}$.

One may also, possibly with further restrictions, extend the analysis of Section 2.3 . 


\section{Correlation with Type Uncertainty}

A restriction in our analysis is that the type uncertainty and residual uncertainty are independently distributed. de Barreda (2011) and Chen (2009) study models where they allow the two types of signals to be correlated. However, these signals do not directly affect the payoff of either player. Since the unconditional variance is at least as large as the mean conditional variance of a correlated signal, there is an intuitive reduction in the "risk" in the distribution of A's type. Interestingly, they show that the endogenously strategic communication could either increase or decrease the effective precision of the communication depending on the parameters.

An analysis of the SITU game while allowing for correlation of different types of uncertainty would require sufficiently different methods and warrants a separate analysis. Nonetheless, it is possible to comment on the issue of risk in the present context through a further consideration of the issues raised in Example 3 involving an uncertain $\mathbf{P}$ versus a privately informed $\mathbf{P}$ in the SITU game. Without accounting for strategic communication, an uncertain $\mathbf{P}$ is clearly subject to greater risk as he is unable to hedge against the residual uncertainty. In Example 3 we saw that this could result in a pooling equilibrium whereas the SITU game admits a fully revealing equilibrium. Interestingly, the reverse could also happen.

Indeed, consider an uncertain $\mathbf{P}$ with slightly more general structure described in Example 1. Now, with an uncertain $\mathbf{P}$ the difference between his ex-post optimal action and that of $\mathbf{A}$, the revised "effective bias" is $b^{\mathfrak{u}}=b+\beta^{p}-\beta^{a}$ whereas the effective bias of the SITU game is simply $b$. Choose any $\ell_{p}, \ell_{a}$ and a distribution of the global parameter $g$ so that Condition $A$ holds and $\beta^{p} \neq \beta^{a}$. If we now select $b=\beta^{a}-\beta^{p}$, then $b^{\mathfrak{u}}=0$, i.e. there is no conflict in the ex-post actions when $\mathbf{P}$ is uncertain. A fully revealing equilibrium can then exist with an uncertain $\mathbf{P}$. However, since $\mathbf{b}^{*}=b \neq 0$, $a$ fully revealing equilibrium cannot exist in the SITU game.

\section{Appendix}

Proof of Lemma 1. First suppose Condition A holds because A has quadratic preferences. Then, $\varphi(x)=\left(x-\mu_{\mathrm{b}}\right)^{2}+\sigma_{\mathrm{b}}^{2}$. Clearly, $\varphi$ achieves a minimum at $\mu_{\mathrm{b}}$ and is symmetric about it.

Now consider the case where Condition A holds because the ex-post bias is symmetrically distributed. Recall that, by definition, a random variable $B$ (with a well-defined mean $\mu_{b}$ ) is symmetric if $B-\mu_{b}$ and $\mu_{b}-B$ have the same probability distribution. Then, for any integrable function, $f, E\left[f\left(B-\mu_{b}\right)\right]=E\left[f\left(\mu_{b}-B\right)\right]$. Using this, and letting 
B denote the random variable of ex-post bias, we have

$$
\begin{aligned}
\varphi\left(\mu_{b}+x\right) & =E\left[\ell_{a}\left(\left|\mu_{b}+x-B\right|\right)\right]=E\left[\ell_{a}\left(\left|x-\left(B-\mu_{b}\right)\right|\right)\right] \\
& =E\left[\ell_{a}\left(\left|x-\left(\mu_{b}-B\right)\right|\right)\right]=E\left[\ell_{a}\left(\left|B+x-\mu_{b}\right|\right)\right] \\
& =E\left[\ell_{a}\left(\left|\mu_{b}-x-B\right|\right)\right]=\varphi\left(\mu_{b}-x\right) .
\end{aligned}
$$

Since $\varphi$ is symmetric about $\mu_{b}$ and it inherits the convexity of $\ell_{a}$, it achieves its unique minimum at $\mu_{b}$, which must be $b^{*}$.

Proof of Lemma 2. Choose any equilibrium strategy profile $\left(\sigma_{a}, \sigma_{p}\right)$. At $\theta, \mathbf{P}$ hears the report $m=\sigma_{a}(\theta)$. The support of his posterior is $\sigma_{a}^{-1}(m)$. His expected loss from selecting an action $\xi^{\prime}$ after observing $\mathbf{s}$ is proportional to

$$
\int_{\theta^{\prime} \in \sigma_{a}^{-1}(m)} \ell_{p}\left(\left|\xi^{\prime}-\theta^{\prime}-w_{p} \cdot s\right|\right) f\left(\theta^{\prime}\right) d \theta^{\prime}
$$

The best-response property requires choosing an action that minimizes the above expression. Now define $\psi$ as follows:

$$
\psi(\theta):=\arg \min _{\xi^{\prime}} \int_{\theta^{\prime} \in \sigma_{\mathrm{a}}^{-1}\left(\sigma_{\mathrm{a}}(\theta)\right)} \ell_{\mathrm{p}}\left(\left|\xi^{\prime}-\theta^{\prime}\right|\right) f\left(\theta^{\prime}\right) \mathrm{d} \theta^{\prime}
$$

Comparing the minimand expression in 19$)$ with the payoff of $\mathbf{P}$ given above, we have $\sigma_{p}(m, s)=\psi(\theta)+\boldsymbol{w}_{p} \cdot \mathbf{s}$.

The proofs of Proposition 2 and Lemma 3 below require the following preliminaries. Fixing $\mathbf{P}$ 's equilibrium behavior and the corresponding $\operatorname{EOF} Y(\theta, \mathbf{s})=\psi(\theta)+\mathbf{w}_{\mathbf{p}} \cdot \mathbf{s}$ (see Lemma 2), the payoff of $\mathbf{A}$ of type $\theta$ from mimicking to be type $\theta^{\prime}$ and sending a signal $\xi^{\prime}=\psi\left(\theta^{\prime}\right)$ is $-\varphi\left(\xi^{\prime}-\theta\right)$.

For any $\xi_{1}=\psi\left(\theta_{1}\right)<\psi\left(\theta_{2}\right)=\xi_{2}$, write

$$
\mathrm{D}\left(\theta, \xi_{1}, \xi_{2}\right)=\frac{\varphi\left(\xi_{2}-\theta\right)-\varphi\left(\xi_{1}-\theta\right)}{\xi_{2}-\xi_{1}} .
$$

Therefore, a type $\theta$ would prefer to mimic being type $\theta_{2}$ instead of type $\theta_{1}$ provided $\left(\xi_{2}-\xi_{1}\right) \mathrm{D}\left(\theta, \xi_{1}, \xi_{2}\right) \leq 0$ and conversely otherwise. $\mathrm{D}\left(\theta, \xi, \xi^{\prime}\right)$ is the slope of $\varphi$ between the points $\xi-\theta$ and $\xi^{\prime}-\theta$. Since $\varphi$ is strictly convex, this slope must be decreasing in $\theta$.

Lemma 3 Consider an equilibrium where $\psi\left(\theta_{1}\right)=\xi_{1}$ and $\psi\left(\theta_{2}\right)=\xi_{2}$ are such that $\xi_{1} \neq \xi_{2}$. Then $\left|\xi_{1}-\xi_{2}\right| \geq\left|b^{*}\right|$.

Proof. Assume, with no loss in generality, that $\xi_{1}<\xi_{2}$. Given P's behavior described 
by Lemma 2, the payoff of a type $\theta$ from reporting $\xi_{i}$ is $-\varphi\left(\xi_{i}-\theta\right)$. By incentive compatibility of equilibrium behavior of $\theta_{1}$, we must have $D\left(\theta_{1}, \xi_{1}, \xi_{2}\right)\left(\xi_{2}-\xi_{1}\right) \geq 0$, and similarly incentive compatibility of $\theta_{2}$ requires $\left(\xi_{2}-\xi_{1}\right) D\left(\theta_{2}, \xi_{1}, \xi_{2}\right) \leq 0$. By continuity of $\mathrm{D}\left(\cdot, \xi_{1}, \xi_{2}\right)$, there must exist some $\theta^{*} \in\left[\theta_{1}, \theta_{2}\right]$ such that $\mathrm{D}\left(\theta^{*}, \xi_{1}, \xi_{2}\right)=0$. By monotonicity, those types to the right of $\theta^{*}$ would strictly prefer to report $\xi_{2}$ and those to its left strictly prefer to report $\xi_{1}$. Therefore, when $\mathbf{P}$ hears $\xi_{1}$ or $\xi_{2}$, he knows the true type is respectively bounded above or below by $\theta^{*}$. Looking at the definition of $\psi$ in $(19)$, we can readily conclude that

$$
\xi_{1} \leq \theta^{*} \leq \xi_{2}
$$

Furthermore, since $\varphi$ is convex with a minimum at $b^{*}$, for $D\left(\theta^{*}, \xi_{1}, \xi_{2}\right)$ to be zero, we must have

$$
\xi_{1}-\theta^{*}<b^{*}<\xi_{2}-\theta^{*} .
$$

Combining (21) and (22), we obtain

$$
\begin{array}{ll}
\xi_{1}<\theta^{*}<\theta^{*}+b^{*}<\xi_{2} & \text { for } b^{*}>0, \\
\xi_{1}<\theta^{*}+b^{*}<\theta^{*}<\xi_{2} & \text { for } b^{*}<0,
\end{array}
$$

implying $\xi_{2}-\xi_{1} \geq\left|\mathrm{b}^{*}\right|>0$. Since $\Theta$ is a compact set, it follows that there can only be finitely many outcomes an equilibrium.

Proof of Proposition 2, Pick an EOF Y. It follows from Lemma 3 and Lemma 2 that in any EOF, there are finitely many values, say $\xi_{1}<\xi_{2}<\cdots<\xi_{N}$, such that $\mathrm{Y}(\theta, \mathbf{s})=\xi_{i}+\boldsymbol{w}_{\mathrm{p}} \cdot \mathbf{s}$ for $1 \leq \boldsymbol{i} \leq \mathbf{N}$. Each $\xi_{i}$ is a possible report that will be sent by some $\theta$ in the equilibrium. We now determine which types send a given report $\xi_{i}$. To this end, with no loss in generality assume that $N \geq 2$ and first define $a_{i}$ by the equation

$$
\varphi\left(\xi_{i}-a_{i}\right)=\varphi\left(\xi_{i+1}-a_{i}\right) \quad \text { for } i=1, \ldots, N-1 \text {. }
$$

Next, recall that the loss of a type $\theta$ from reporting $\xi_{i}$, given the behavior of $\mathbf{P}$, is $\varphi\left(\xi_{i}-\theta\right)$ and hence $\left(\xi_{i+1}-\xi_{i}\right) \mathrm{D}\left(\theta, \xi_{i}, \xi_{i+1}\right)$ is the difference in type $\theta$ 's payoff from reporting $\xi_{i+1}$ instead of $\xi_{i}$. Since $D\left(\cdot, \xi_{i}, \xi_{i+1}\right)$ is decreasing, all the types in $\left[\theta_{\ell}, a_{i}\right)$ would strictly prefer reporting $\xi_{i}$ instead of $\xi_{i+1}$ whereas the opposite is true for types in $\left(a_{i}, \theta_{h}\right]$ and type $a_{i}$, by its definition above, is indifferent between either report. Consequently $\left(a_{i-1}, a_{i}\right)$ is the set of types that strictly prefer to report $\xi_{i}$ to any of the other reports. Type $a_{i}$ is indifferent between reporting $\xi_{i}$ and $\xi_{i+1}$, and strictly prefers those over any other 
report. Therefore, 19) reduces to

$$
\xi_{i}=\arg \min _{\xi} \int_{a_{i-1}}^{a_{i}} \ell_{p}(\xi-\theta) f(\theta) d \theta \quad \forall i=1, \ldots, N .
$$

Noting that $\xi_{i}=\chi\left(a_{i-1}, a_{i}\right)$ completes the proof of Part 1 of the Proposition. For the proof of Part 2 of the Proposition, one need only follow the arguments given in the proof of Theorem 1 in Crawford and Sobel (1982) 14

Proof of Proposition 3. Given the discussion that followed the Proposition, we need only prove Part 2 . Let $[\alpha, \beta]$ denote a bound for the support of the ex-post bias. If $\boldsymbol{a}$ is the partition in the $\mathrm{N}$-equilibrium, letting $x_{i}:=x\left(a_{i-1}, a_{i}\right)$, the ex-ante payoff of $\mathbf{A}$ in this equilibrium is

$$
\pi_{N}^{a}(\mathbf{a}, H)=-\sum_{i=1}^{N} \int_{a_{i-1}}^{a_{i}} E_{H}\left[\ell_{a}\left(\left|x_{i}-\theta-B\right|\right)\right] d F(\theta),
$$

where $\mathrm{B}$ denotes the random variable of the ex-post bias, $\mathrm{H}$ denotes its distribution and

$$
E_{H}\left[\ell_{a}(|x-B|)\right]=\int_{\alpha}^{\beta} \ell_{a}(|x-b|) d H(b) .
$$

Now consider a change in the distribution of the ex-post bias to $\mathrm{H}^{\prime}$ so that it second order stochastically dominates $\mathrm{H}$ and the mean remains unchanged. An unchanged mean implies

$$
\int_{\alpha}^{\beta} H(x) d x=\int_{\alpha}^{\beta} H^{\prime}(x) d x
$$

Given that $\ell_{a}$ is convex, a direct application of the proof of Theorem $3^{\prime}$ of Hadar and Russell (1969) together with the above equality immediately gives us

$$
E_{H}\left(\ell_{a}(|x-B|)\right)>E_{H^{\prime}}\left(\ell_{a}(|x-B|)\right) .
$$

Since the mean has not changed, the $\mathrm{N}$-equilibrium partition remains unchanged under $\mathrm{H}^{\prime}$ and the payoff of $\mathbf{A}$ after the change is $\pi_{\mathrm{N}}^{\mathrm{a}}\left(\mathbf{a}, \mathrm{H}^{\prime}\right)$ leading to the conclusion that $\mathbf{A}$ is better off from the change. Since the payoff of $\mathbf{P}$ only depends on the mean bias, his payoff is unchanged.

Proof of Proposition 4. Simplify the notation by setting $\pi_{N}\left(\mu_{b}\right)=\pi_{N}(b)$. We will routinely appeal to the following comparative statics:

\footnotetext{
${ }^{14}$ Repeat the proof arguments given on Para 2, Page 1438 onwards replacing their condition (A) with (23) and their Eq. (10) with (24) above.
} 


$$
\begin{aligned}
& \pi_{N-1}(b)<\pi_{N}(b) \text { whenever } \quad N \leq N_{b}^{*} \\
& N_{b}^{*} \leq N_{b^{\prime}}^{*} \text { whenever }\left|b^{\prime}\right|<|b| \\
& \pi_{N}(b)<\pi_{N}\left(b^{\prime}\right) \quad \text { whenever } \quad\left|b^{\prime}\right|<|b|
\end{aligned}
$$

Assertion CS1 is Part 2, Corollary 1 while CS2 and CS3 are Part 1, Proposition 3.

We will first establish two auxiliary results, Lemma 4 and Lemma 5 . Lemma 4 considers what payoffs $\mathbf{P}$ can achieve in a CS-game with constant bias if his information about $\theta$ is not constrained by strategic considerations but instead he could choose any partition of $\Theta$ into $\mathrm{N}$ sub-intervals. Lemma 5 then relates these payoffs in an $\mathrm{N}$-equilibrium when the bias is small.

Let $\Delta_{\mathrm{N}}$ represent all possible ways of dividing $\Theta$ into at most $\mathrm{N}$ sub-intervals. That is, setting $a_{0}:=\theta_{\ell}$,

$$
\Delta_{N}=\left\{\left(a_{1}, \ldots, a_{N}\right) \in \Theta^{N}: a_{i-1} \leq a_{i}, \quad i=1, \ldots, N \quad \text { and } \quad a_{N}=\theta_{h}\right\}
$$

Given $\mathbf{a}_{N} \in \Delta_{N}$ and any vector $\mathbf{x}=\left(\xi_{1}, \ldots, \xi_{N}\right) \in \mathbb{R}^{N}$, let

$$
\Pi_{N}\left(\mathbf{x}, \mathbf{a}_{N}\right)=-\sum_{k=1}^{N} \int_{a_{k-1}}^{a_{k}} \ell_{p}\left(\left|\xi_{k}-\theta\right|\right) f(\theta) d \theta
$$

$\Pi_{N}\left(\mathbf{x}, \mathbf{a}_{N}\right)$ is the ex-ante payoff of $\mathbf{P}$ if his information upon realization of $\theta$ is given by $\mathbf{a}_{N}$ and he chooses according to $\mathbf{x}$. Since $\ell_{p}$ is continuous and convex, and $\Delta_{N}$ is compact, each of the following is well-defined:

$$
h_{N}\left(\mathbf{a}_{N}\right):=\arg \max _{\mathbf{x}} \Pi_{N}\left(\mathbf{x}, \mathbf{a}_{N}\right), \quad \mathbf{a}_{N}^{*}:=\arg \max _{\mathbf{a}_{N} \in \Delta_{N}} \Pi_{N}\left(h\left(\mathbf{a}_{N}\right), \mathbf{a}_{N}\right)
$$

Set $\Pi_{N}\left(h\left(\mathbf{a}_{N}^{*}\right), \mathbf{a}_{N}^{*}\right)=\Pi_{N}^{*}$. In words, if $\mathbf{P}$ were given the option of dividing $\Theta$ into at most $\mathrm{N}$ sub-intervals with the knowledge that it would be revealed later which of these sub-intervals that $\theta$ lies in, he would cut $\Theta$ according to $\mathbf{a}_{\mathrm{N}}^{*}$.

Lemma $4 \lim _{\mathrm{N} \rightarrow \infty} \Pi_{\mathrm{N}}^{*}=-\ell_{\mathrm{p}}(0)$.

Proof. Let $\Pi_{N}\left(\mathbf{a}_{N}\right)=\Pi_{N}\left(h_{N}\left(\mathbf{a}_{N}\right), \mathbf{a}_{n}\right)$. Since, $\ell_{p}$ is uniformly continuous on $\Theta$, for any $\epsilon>0$, there exists a $\delta>0$ such that $\ell_{p}(|\xi|)<\ell_{p}(0)+\epsilon$ whenever $|\xi|<\delta$. Consequently, for any partition $\mathbf{a}_{N}$ with a norm $\left\|\mathbf{a}_{N}\right\|<\delta$, we must have $\max _{\xi} \int_{a_{i-1}}^{a_{i}} \ell_{p}(|\xi-\theta|) f(\theta) \mathrm{d} \theta<$ $\left(F\left(a_{i}\right)-F\left(a_{i-1}\right)\right)\left(\ell_{p}(0)+\epsilon\right)$ and hence $\Pi_{N}\left(a_{N}\right)>-\ell_{p}(0)-\epsilon$. Further, since $\ell_{p}(|\xi|) \geq \ell_{p}(0)$ for all $\xi$, we also have $\Pi_{N}\left(\mathbf{a}_{N}\right) \leq-\ell_{p}(0)$. In other words, for any sequence of partitions $\left(\mathbf{a}_{N}\right),\left\|\mathbf{a}_{N}\right\| \rightarrow 0$ implies $\Pi_{N}\left(\mathbf{a}_{N}\right) \rightarrow \ell_{p}(0)$. Therefore, the proof is complete on showing that $\lim _{\mathrm{N} \rightarrow \infty}\left\|\mathbf{a}_{\mathrm{N}}^{*}\right\|=0$. 
Given any $\alpha<\xi<\beta$, define

$$
\mathrm{L}(\xi, \alpha, \beta)=\min _{\xi_{1}} \int_{\alpha}^{\xi} \ell_{p}\left(\left|\xi_{1}-\theta\right|\right) f(\theta) \mathrm{d} \theta+\min _{\xi_{2}} \int_{\xi}^{\beta} \ell_{p}\left(\left|\xi_{2}-\theta\right|\right) f(\theta) \mathrm{d} \theta
$$

Observe that $\mathrm{L}$ is continuous and for a fixed $\xi$, it is strictly decreasing in $\alpha$ and strictly increasing in $\beta$. Therefore, given any $\varepsilon>0$, define

$$
\delta(\varepsilon, \xi)=\max _{\theta_{\ell} \leq \alpha \leq \xi-\varepsilon, \xi+\varepsilon \leq \beta \leq \theta_{h}}(\mathrm{~L}(\xi, \alpha, \beta)-\mathrm{L}(\alpha, \alpha, \beta)) .
$$

Observe that $\mathrm{L}(\xi, \alpha, \beta)<\mathrm{L}(\alpha, \alpha, \beta)$ for any such $\alpha, \xi, \beta$ and hence $\delta(\varepsilon, \xi)<0$.

Now suppose, by way of contradiction, that $\lim _{N \rightarrow \infty}\left\|\mathbf{a}_{N}^{*}\right\|>0$. It follows that there must exist a $\xi$ and $\varepsilon>0$ such that the interval $[\xi-\varepsilon, \xi+\varepsilon]$ is contained in some subinterval of $\mathbf{a}_{\mathrm{N}}^{*}$, say $\left[\mathrm{a}_{\mathrm{k}-1}^{*}, \mathbf{a}_{\mathrm{k}}^{*}\right.$. Now cut this interval in two at $\xi$ and let $\hat{\mathbf{a}}_{\mathrm{N}}^{*}$ denote the obvious partition of $\mathrm{N}+1$ sub-intervals obtained from $\mathbf{a}_{\mathrm{N}}^{*}$. Then,

$$
\begin{aligned}
\Pi_{N}^{*}=\Pi_{N}\left(\mathbf{a}_{N}^{*}\right) & =-\sum_{i=1}^{k-1} L\left(a_{i-1}^{*}, a_{i-1}^{*}, a_{i}^{*}\right)-\sum_{i=k+1}^{N} L\left(a_{i-1}^{*}, a_{i-1}^{*}, a_{i}^{*}\right) \\
& -L\left(a_{k-1}^{*}, a_{k-1}^{*}, a_{k}^{*}\right) \\
\leq & -\sum_{i=1}^{k-1} L\left(a_{i-1}^{*}, a_{i-1}^{*}, a_{i}^{*}\right)-\sum_{i=k+1}^{N} L\left(a_{i-1}^{*}, a_{i-1}^{*}, a_{i}^{*}\right) \\
& -L\left(\xi, a_{k-1}^{*}, a_{k}^{*}\right)+\delta(\varepsilon, \xi) \\
& =\Pi_{N+1}\left(\hat{a}_{N+1}^{*}\right)+\delta(\varepsilon, \xi) \\
\leq & \Pi_{N+1}^{*}+\delta(\varepsilon, \xi) .
\end{aligned}
$$

Note that $\left(\Pi_{N}^{*}\right)$ is a non-decreasing sequence bounded above by $-\ell_{p}(0)$ and hence its limit exists. Taking the limit as $\mathrm{N} \rightarrow \infty$ on both sides of the above inequality, we readily arrive at the contradiction that $\delta(\varepsilon, \xi)<0$.

Lemma 5 Consider the family of CS-game(s) with a fixed bias, indexed by the bias $\mathrm{b}$, in which $\ell_{\mathrm{p}}$ is the loss function of $\boldsymbol{P}$. Then,

$$
\lim _{b \rightarrow 0} \pi_{N}(b)=\Pi_{N}^{*}
$$

Furthermore, given any integer $\mathrm{N}$, for all b sufficiently close to zero, an $\mathrm{N}$-equilibrium of the corresponding CS game with the bias b exists.

Proof. The $\mathrm{N}$-equilibrium of the CS-game with a fixed bias 0 , if it exists, is fully characterized by the partition $\mathbf{a}^{0}=\left(a_{1}^{0}, \ldots, a_{N}^{0}\right)$ given as the solution to the following 
system of equations for all $i=1, \ldots, N-1$ :

$$
\begin{aligned}
& \xi_{i}^{0}=\arg \min _{\xi} \int_{a_{i-1}^{0}}^{a_{i}^{0}} \ell_{p}(|\xi-\theta|) f(\theta) d \theta, \\
& a_{i}^{0}=\frac{\xi_{i}^{0}+\xi_{i+1}^{0}}{2} .
\end{aligned}
$$

Now let $h_{N}\left(\mathbf{a}_{N}^{*}\right)=\left(\xi_{1}^{*}, \ldots, \xi_{N}^{*}\right)$. Given the definition of $\mathbf{a}_{N}^{*}$ (in $(30)$ ), upon applying the envelope theorem, we have

$$
\begin{aligned}
& \xi_{i}^{*}=\arg \min _{\xi} \int_{a_{i-1}^{*}}^{a_{i}^{*}} \ell_{p}(|\xi-\theta|) f(\theta) d \theta, \\
& a_{i}^{*}=\frac{\xi_{i}^{*}+\xi_{i+1}^{*}}{2},
\end{aligned}
$$

where (36) is merely the first-order condition for maximizing $\Pi_{N}\left(h\left(\mathbf{a}_{N}\right), \mathbf{a}_{N}\right)$ with respect to $\mathbf{a}_{\mathrm{N}}$. In addition, following Theorem 2 of CS, for any $\mathrm{N}$, there is at most one solution satisfying (33) and (34). This implies $\mathbf{a}_{N}^{*}=\mathbf{a}_{N}^{0}$ and hence $\pi_{N}(0)=\Pi_{N}^{*}$. Hence an $\mathrm{N}$-equilibrium exists when the bias is zero. Comparing $\mathbf{a}_{N}^{b}$, the equilibrium partition described in Corollary 1, with (33) and (34), it is clear that $\mathbf{a}_{\mathrm{N}}^{\mathrm{b}} \approx \mathbf{a}_{\mathrm{N}}^{0}$ when $\mathbf{b} \approx 0$. The Lemma now follows from these observations and CS2.

Completing the Proof of Proposition 4. Part 1 of the Proposition follows from Lemma 5. To prove Part 2, let $\left(\mathrm{N}_{\mathrm{k}}\right)$ and $\left(\mathrm{b}_{\mathrm{k}}\right)$ be as given in the Proposition. For any integer $m$, define

$$
\mathrm{n}_{\mathrm{m}}=\inf _{\mathrm{k} \geq \mathrm{m}} \mathrm{N}_{\mathrm{k}}
$$

Since $n_{m} \leq N_{k} \leq N_{b_{k}}^{*}$ for all $k \geq m$, we know that for all such $k$, an $n_{m}$-equilibrium exists when the bias is $b_{k}$ with the property that

$$
\begin{aligned}
\pi_{\mathrm{N}_{k}}\left(b_{k}\right) & \geq \pi_{\mathrm{n}_{m}}\left(b_{k}\right) \quad(\text { by CS1), } \\
\text { and therefore } \quad \liminf _{k \rightarrow \infty} \pi_{N_{k}}\left(b_{k}\right) & \geq \Pi_{n_{m}}^{*} \quad(\text { by Lemma 5). }
\end{aligned}
$$

Now take the limit as $m \rightarrow \infty$ in the above inequality to deduce from Lemma 4 that the above RHS converges to $-\ell_{p}(0)$ and the result then follows using $\pi_{N_{k}}\left(b_{k}\right) \leq-\ell_{p}(0)$.

Proof of Proposition 6. By Corollary 1, the payoff from retaining authority and playing the SITU game is the same as $\mathbf{P}$ 's payoff in the CS-game with quadratic loss functions and bias $\mu_{\mathrm{b}}$. In an $\mathbf{N}$-equilibrium of the latter, the expected loss of $\mathbf{P}$ is simply the residual variance of $\theta$ that $\mathbf{P}$ expects after hearing $\mathbf{A}$ 's report. That is, if $\xi_{N}^{*}: \Theta \longrightarrow \mathbb{R}$ 
denotes the equilibrium outcome function of the CS-game, then P's loss from retaining authority, as shown in Section 4 of CS with the bias $b=\mu_{b}$, is

$$
E\left[\ell_{p}\left(\xi_{N}(\theta)\right)\right]=\frac{1}{12 N^{2}}+\mu_{b}^{2} \frac{\left(N^{2}-1\right)}{3} .
$$

On the other hand, if $\mathbf{P}$ delegates, $\mathbf{A}$ chooses the action $\theta+\boldsymbol{w}_{\mathrm{a}} \cdot \mathbf{s}$ in state $(\theta, \mathbf{s})$ leaving $\mathbf{P}$ with an ex-post payoff of $\ell_{\mathfrak{p}}\left(b_{\mathbf{s}}\right)$. Given that $\ell_{\mathfrak{p}}$ is assumed to be quadratic, the loss from delegation is $\mu_{\mathrm{b}}^{2}+\sigma_{\mathrm{b}}^{2}$. Retaining authority is therefore a superior choice whenever

$$
\begin{aligned}
\mu_{\mathrm{b}}^{2}+\sigma_{\mathrm{b}}^{2} & >\frac{1}{12 \mathrm{~N}^{2}}+\mu_{\mathrm{b}}^{2} \frac{\left(\mathrm{N}^{2}-1\right)}{3} \\
\Leftrightarrow \quad \sigma_{\mathrm{b}}^{2} & >\frac{1}{12 \mathrm{~N}^{2}}+\mu_{\mathrm{b}}^{2}\left(\frac{\left(\mathrm{N}^{2}-4\right)}{3}\right) .
\end{aligned}
$$

(40) establishes Part 1. For Part 2, recall that CS show that the existence of an equilibrium which divides $\Theta$ into $\mathrm{N}$ sub-intervals requires that

$$
\mathrm{N} \leq-\frac{1}{2}+\frac{1}{2}\left(1+\frac{2}{\mu_{\mathrm{b}}}\right)^{1 / 2} \quad \text { or equivalently, } \quad \mu_{\mathrm{b}} \leq \frac{2}{(2 \mathrm{~N}+1)^{2}-1}
$$

Therefore, the RHS of 40 is bounded above by

$$
\frac{1}{12 N^{2}}+\left(\frac{2}{(2 N+1)^{2}-1}\right)^{2} \frac{\left(N^{2}-4\right)}{3}=\frac{\left(2 N^{2}+2 N-3\right)}{N^{2}(N+1)^{2}} \sigma_{\theta}^{2}
$$

Hence, 15 implies 40 and Part 2 follows.

Proof of Proposition 7. The proof uses the comparative statics CS1, CS2 and CS3 listed in the proof of Proposition 4 as well as Proposition 4 itself. Under the given hypothesis, applying $(12)$, the payoff from delegation is at most $-K \epsilon$. Let $b_{\epsilon}=\sup \{|b|$ : $\left.\pi_{N_{b}^{*}}(b) \geq-K \epsilon\right\}$. Note that $b_{\epsilon}$ is well-defined, due to Proposition 4 . Define $N_{\epsilon}=N_{b_{\epsilon}}^{*}+1$. For the existence of an $N$-equilibrium, where $N \geq N_{\epsilon}$, we must have $\left|\mu_{b}\right|<\left|b_{\epsilon}\right|$ and by construction then $\pi_{N}\left(\mu_{b}\right)>-K \epsilon$.

\section{References}

Aghion, Philippe and Jean Tirole, "Formal and Real Authority in Organizations," Journal of Political Economy, 1997, 105 (1), 1-29.

Alonso, Ricardo and Niko Matouschek, "Relational Delegation," Rand Journal of Economics, 2007, 38 (4), 1070-1089.

_ and _, "Optimal Delegation," Review of Economic Studies, 2008, 75, 259-293. 
Ambrus, Attila and Satoru Takahashi, "Multi-sender Cheap Talk with Restricted State Spaces," Theoretical Economics, 2008, 3 (1), 1-27.

Armstrong, Mark, "Delegation and Discretion," Working paper 1994.

- and John Vickers, "A Model of Delegated Project Choice," Econometrica, 2010, 78 (1), 213-244.

Battaglini, Marco, "Multiple Referrals and Multidimensional Cheap Talk," Econometrica, 2002, 70 (4), 1379-1401.

Blume, Andreas, Oliver J. Board, and Kohei Kawamura, "Noisy Talk," Theoretical Economics, 2007, 2 (4), 395-440.

Chakraborty, Archishman and Rick Harbaugh, "Persuasion by Cheap Talk," American Economic Review, 2010, 100 (5), 2361-82.

Chen, Ying, "Communication with Two-Sided Asymmetric Information," Working paper, Department of Economics, Arizona State University 2009.

_ , Navin Kartik, and Joel Sobel, "Selecting Cheap-Talk Equilibria," Econometrica, 2008, 76 (1), 117-136.

Crawford, Vincent P. and Joel Sobel, "Strategic Information Transmission," Econometrica, 1982, 50 (6), 1431-51.

de Barreda, Ines Moreno, "Cheap Talk with Two-Sided Private Information," Working paper, Department of Economics, London School of Economics 2011.

Dessein, Wouter, "Authority and Communication in Organizations," Review of Economic Studies, 2002, 69 (4), 811-38.

Fudenberg, Drew and David Levine, Game Theory, The MIT Press, 1990.

Gilligan, Thomas W. and Keith Krehbiel, "Asymmetric Information and Legislative Rules with a Heterogeneous Committee," American Journal of Political Science, 1989, 33, 459-490.

Goltsman, Maria and Gregory Pavlov, "How to Talk to Multiple Audiences," Games and Economic Behavior, 2011, 72 (1), 100-122.

Hadar, Josef and William R Russell, "Rules for Ordering Uncertain Prospects," American Economic Review, March 1969, 59 (1), 25-34.

Harris, Milton and Artur Raviv, "Allocation of Decision-making Authority," Review of Finance, 2005, 9 (3), 353-383. 
Holmstrom, Bengt, "On the Theory of Delegation," in M. Boyer and R.E. Kihlstrom, eds., Bayesian Models in Economic Theory, New York: North-Holland, 1984.

Kartik, Navin, "Strategic Communication with Lying Costs," Review of Economic Studies, 2009, 76 (4), 1359-1395.

_ , Marco Ottaviani, and Francesco Squintani, "Credulity, Lies, and Costly Talk," Journal of Economic Theory, 2007, 134 (1), 93-116.

Koessler, Frederic and David Martimort, "Optimal Delegation with Multidimensional Decisions," Working paper, 2010.

Krahmer, Daniel, "Message-contingent Delegation," Journal of Economic Behavior and Organization, 2006, 60, 490-506.

Krishna, Vijay and John Morgan, "A Model Of Expertise," The Quarterly Journal of Economics, 2001, 116 (2), 747-775.

_ and _ , "The Art of Conversation: Eliciting Information from Experts through Multistage Communication," Journal of Economic Theory, 2004, 117 (2), 147-179.

Levy, Gilat and Ronny Razin, "On the Limits of Communication in Multidimensional Cheap Talk: A Comment," Econometrica, 2007, 75 (3), 885-893.

Li, Ming and Kristf Madarsz, "When Mandatory Disclosure Hurts: Expert Advice and Conflicting Interests," Journal of Economic Theory, 2008, 139 (1), 47-74.

McGee, Andrew, "Delegation and Consultation with Contingent Information," Working Paper, Department of Economics, Ohio State University 2009.

Melumad, Nahum D. and Toshiyuki Shibano, "Communication in Settings with No Transfers," Rand Journal of Economics, 1991, 22 (2), 173-198.

Myerson, Roger, Game Theory: Analysis of Conflict, Harvard University Press, 1991.

Ottaviani, Marco, "The Economics of Advice," Working paper 2000.

Seidmann, Daniel J., "Effective Cheap Talk with Conflicting Preferences," Journal of Economic Theory, 1990, 50 (2), 445-458.

- and Eyal Winter, "Strategic Information Transmission with Verifiable Messages," Econometrica, 1997, 65 (1), 163-170.

Spector, David, "Pure Communication between Agents with Close Preferences," Economics Letters, 2000, 66 (2), 171-178. 
Watson, Joel, "Information Transmission When the Informed Party is Confused," Games and Economic Behavior, 1996, 12 (1), 143-161.

Wolinsky, Asher, "Eliciting Information from Multiple Experts," Games and Economic Behavior, 2002, 41 (1), 141-160. 